PHYSICAL REVIEW B 87, 174513 (2013)

\title{
Quantum phase slips in Josephson junction rings
}

\author{
G. Rastelli,, ${ }^{1,2}$ I. M. Pop, ${ }^{3,4}$ and F. W. J. Hekking ${ }^{1}$ \\ ${ }^{1}$ Université Grenoble 1/CNRS, LPMMC UMR 5493, B.P. 166, 38042 Grenoble, France \\ ${ }^{2}$ Fachbereich Physik, Universität Konstanz, D-78457 Konstanz, Germany \\ ${ }^{3}$ Institut Néel, CNRS, and Université Joseph Fourier, B.P. 166, 38042 Grenoble, France \\ ${ }^{4}$ Department of Applied Physics, Yale University, New Haven, Connecticut 06511, USA \\ (Received 6 January 2012; revised manuscript received 25 April 2013; published 16 May 2013)
}

\begin{abstract}
We study quantum phase-slip (QPS) processes in a superconducting ring containing $N$ Josephson junctions and threaded by an external static magnetic flux $\Phi_{B}$. In such a system, a QPS consists of a quantum tunneling event connecting two distinct classical states of the phases with different persistent currents [Matveev et al., Phys. Rev. Lett. 89, 096802 (2002)]. When the Josephson coupling energy $E_{J}$ of the junctions is larger than the charging energy $E_{C}=e^{2} / 2 C$, where $C$ is the junction capacitance, the quantum amplitude for the QPS process is exponentially small in the ratio $E_{J} / E_{C}$. At given magnetic flux, each QPS can be described as the tunneling of the phase difference of a single junction of almost $2 \pi$, accompanied by a small harmonic displacement of the phase difference of the other $N-1$ junctions. As a consequence, the total QPS amplitude $v_{\text {ring }}$ is a global property of the ring. Here, we study the dependence of $v_{\text {ring }}$ on the ring size $N$, taking into account the effect of a finite capacitance $C_{0}$ to ground, which leads to the appearance of low-frequency dispersive modes. Josephson and charging effects compete and lead to a nonmonotonic dependence of the ring's critical current on $N$. For $N \rightarrow \infty$, the system converges either towards a superconducting or an insulating state, depending on the ratio between the charging energy $E_{0}=e^{2} / 2 C_{0}$ and the Josephson coupling energy $E_{J}$.
\end{abstract}

DOI: 10.1103/PhysRevB.87.174513

PACS number(s): 74.50.+r, 74.81.Fa, 73.23.Ra, 85.25.Cp

\section{INTRODUCTION}

One-dimensional Josephson junction chains (1D JJ chains) have received considerable interest recently. Their use has been proposed for the realization of a qubit topologically protected against decoherence, ${ }^{1-6}$ for the realization of a tunable parametric amplifier in narrow frequency ranges, ${ }^{7,8}$ for the realization of a fundamental current standard in quantum metrology, ${ }^{9,10}$ and for designing controlled inductive electromagnetic environments in quantum circuitry. ${ }^{11,12}$

Homogeneous JJ chains of infinite length have been studied theoretically in the past. ${ }^{13-18}$ Such chains consist of superconducting islands, separated by Josephson tunnel junctions. In this paper, we consider JJ chains arranged in a closed geometry (Fig. 1). The electrostatic interaction between the metallic islands is modeled by a neighboring capacitance $C$ and by a local ground capacitance $C_{0}$, with $E_{C}=e^{2} / 2 C$ and $E_{0}=e^{2} / 2 C_{0}$ the corresponding charging energies. Each Josephson junction can sustain a maximum supercurrent $I_{J}=2 e E_{J} / \hbar$; this defines the Josephson coupling energy $E_{J}$.

Previous theoretical studies ${ }^{13-15}$ predicted a superconductor-insulator phase transition when the ratio between the Josephson energy $E_{J}$ and the characteristic charging energy is reduced below a critical value. Bradley and Doniach studied this phase transition for infinite $\mathrm{JJ}$ chains and for the two extreme opposite cases when one of the two capacitances is vanishing $\left(C_{0}=0\right.$ or $\left.C=0\right) \cdot{ }^{13}$ Korshunov investigated the general case for arbitrary ratio $C / C_{0} \cdot{ }^{14,15} \mathrm{He}$ found that the critical value $E_{J}^{(c)}$ of the Josephson energy at which the system undergoes the phase transition equals to $E_{J}^{(c)}=E_{0} f\left(C / C_{0}\right)$ where $f(x)$ is a smooth and regular function of order one. In particular, for the case $C_{0}=0$, we have $E_{0}=\infty=E_{J}^{(c)}$ and the system is an insulator for any value of $E_{J}$ in agreement with the result of Bradley and Doniach. ${ }^{13}$ Subsequently, experimental studies of the finite-temperature behavior of the residual resistance in long one-dimensional chains of SQUIDs reported a phase transition when reducing the Josephson energy. ${ }^{19-23}$

The theoretical results reviewed so far were obtained in the thermodynamic limit $N \rightarrow \infty$. A first attempt to go beyond this limit was undertaken by Matveev, Glazman, and Larkin $^{24}$ who studied quantum phase-slip (QPS) processes in a superconducting nanoring containing a large, but finite number of Josephson junctions. Here, a QPS consists of a quantum tunneling between two distinct classical states of the phases with different persistent currents circulating in the ring at given magnetic flux. ${ }^{24}$ This is a collective process which can be described as the tunneling of the phase difference of a single junction by almost $2 \pi$, accompanied by a small harmonic displacement of the phase difference of the other $N-1$ junctions (see Ref. 24 and the explanation in Sec. III B). Quantum tunneling is possible due to the finite junction capacitance, which plays the role of inertia.

Matveev et al. predicted a strong reduction of the maximum critical current sustained by the ring with increasing ring size $N$ due to QPS processes. Recent experiments on flux-biased rings containing a few Josephson junctions ${ }^{25}$ indeed reported a remarkable suppression of the maximum supercurrent as $E_{J} / E_{C}$ decreases, in agreement with the findings of Ref. 24. In these devices, the effects of the capacitance to ground could be neglected since the ring's circumference was much smaller than the screening length $\lambda$ of the system, given by $\lambda=\pi \sqrt{C / C_{0}}$. However, it is expected that, for $\mathrm{JJ}$ rings of intermediate circumference $N \gtrsim \lambda$, the effects of the capacitance to ground can no longer be ignored.

In this paper, we study a JJ ring of finite circumference and threaded by an external magnetic flux $\Phi_{B}$ (Fig. 1). Specifically, we consider properties of the flux-dependent thermodynamic persistent current. We go beyond the previous work of Matveev et al. ${ }^{24}$ and we take into account the collective nature of a QPS as well as the ground capacitance $C_{0}$ for calculating 


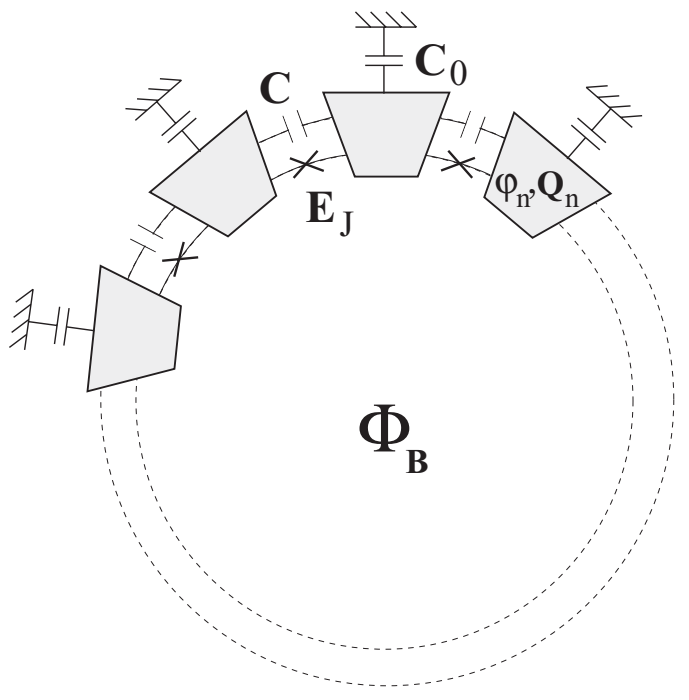

FIG. 1. Schematic representation of a superconducting ring threaded by a magnetic flux $\Phi_{B}$ and containing $N$ identical Josephson junctions with a mutual capacitance $C$ and a local ground capacitance $C_{0} . E_{J}$ is the energy scale for the Josephson coupling. The variables $\left(\varphi_{n}, Q_{n}\right)$ are, respectively, the condensate phase and the excess charge of the $n$th superconducting island.

the QPS amplitude. We show that the interplay between the finite value of the ratio $C_{0} / C$ and finite-size effects gives rise to a nonmonotonic dependence of the low-energy properties on $N$. We systematically discuss this interplay as well as its consequences for the QPS amplitude for flux-biased rings with arbitrary number $N \gtrsim 5$. For shorter lengths, a detailed numerical analysis was realized in Ref. 26.

We focus on the limit where the Josephson coupling energy $E_{J}$ dominates over the charging energies $E_{C}, E_{0}$, such that the amplitude for QPS to occur is exponentially small in the ratio $E_{J} / E_{C}$. This fact allows us to focus on the analysis of a single QPS event. Once the QPS amplitude is known, one can calculate the ring's low-energy spectrum as a function of the external flux $\Phi_{B}$ and hence obtain the maximum supercurrent $I_{\max }$ that the ring can sustain.

\section{QUALITATIVE DISCUSSION AND MAIN RESULTS}

Our main results are summarized in Fig. 2, where we show the dependence of $I_{\max }$, scaled to the classical value $I_{\mathrm{cl}}=$ $\pi I_{J} / N$ found in the absence of QPS processes, as a function of $N$ for two relevant situations: $C_{0}=0$ and $C / 2$.
As we will discuss in detail in the following, a QPS can be described as a $2 \pi(1-1 / N)$ winding of the local phase difference occurring on one of the junctions, accompanied by a simultaneous small (harmonic) adjustment of the phases of the other $N-1$ junctions. ${ }^{24}$ In a first approximation, the winding of the phase on one of the junctions can be characterized by the amplitude for the quantum tunneling between two different minima of the Josephson potential. It is given by ${ }^{24,27-29}$

$$
\nu_{0}=\frac{4}{\sqrt{\pi}}\left(8 E_{J}^{3} E_{C}\right)^{\frac{1}{4}} \exp \left(-\sqrt{8 \frac{E_{J}}{E_{C}}}\right) .
$$

The dynamics of the simultaneous small adjustment depends crucially on the capacitance ratio $C_{0} / C$.

Consider first the case $C_{0}=0$ [Fig. 2(a)]. The other $N-1$ junctions form a bath of dispersionless harmonic oscillators, all having the same (plasma) frequency $\omega_{p}=\left(8 E_{J} E_{C}\right)^{1 / 2} / \hbar$. In order to satisfy the constraint imposed by the flux threading the ring at all times during the QPS process, the phase differences for the $N-1$ other junctions perform a small shift $\sim 1 / N$. This adjustment gives rise to finite-size corrections for intermediate ring circumferences $N$ to the amplitude given by Eq. (1) leading to a QPS amplitude $v_{0} \rightarrow v(N)$. Since any junction can act as a QPS center, the total QPS amplitude for the ring is given by $v_{\text {ring }}=N v(N)$.

In the limit $N \gg 1$, finite-size effects vanish and $v(N)$ converges to the constant $\nu_{0}$ so that $\nu_{\text {ring }}$ increases with the length and the maximum supercurrent $I_{\max }$ vanishes exponentially ${ }^{24}$ [see Fig. 2(a)]. The system becomes a perfect insulator at $N=\infty$, in agreement with Refs. 13-15. On the other hand, we find that the interplay between charging and Josephson effects in finite systems leads to an enhancement of the effective QPS amplitude $v(N)$ with decreasing ring circumference $N$, $v(N) \sim v_{0} \exp \left[\left(E_{J} / E_{C}\right)^{1 / 2} / N\right]$, thus reducing the maximum supercurrent $I_{\max }$. Consequently, the maximum supercurrent $I_{\max }$ shows nonmonotonic behavior as a function of the ring circumference $N$ for sufficiently large values of the ratio $E_{J} / E_{C}$.

When the capacitance to ground $C_{0}$ is restored, the $N-1$ harmonic junctions interact directly between them. This leads to the appearance of an ensemble of $N-1$ dispersive electrodynamics modes at frequencies below $\omega_{p}$, similar to the ones found in a standard LC-transmission line. ${ }^{30,31}$ The tunneling phase couples to these modes in much the same way as a quantum particle to a harmonic bath in the CaldeiraLeggett model. ${ }^{32}$ In particular, the low-frequency modes with linear dispersion $\hbar \omega_{k} \sim\left(8 E_{J} E_{0}\right)^{1 / 2} \pi k / N$ give rise to a finite friction for the QPS dynamics in the limit $N=\infty$. (a)

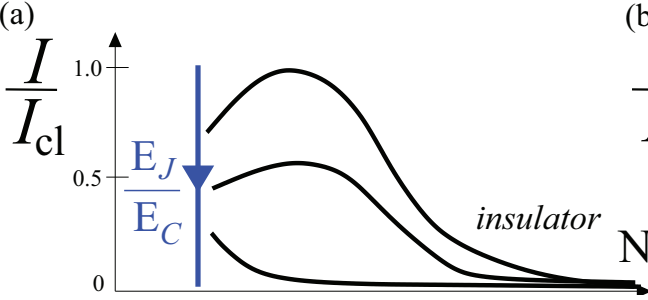

(b)

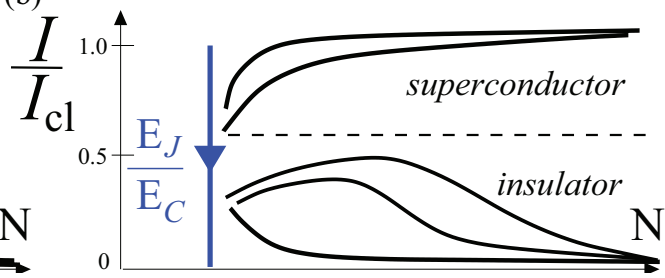

FIG. 2. (Color online) Schematic behavior of the maximum supercurrent $I_{\max }$ in $1 \mathrm{D} \mathrm{JJ}$ rings as a function of $N$, scaled to the classical value $I_{\mathrm{cl}}$, for different values of the ratio $E_{J} / E_{C}$ for (a) $C_{0}=0$ and (b) $C_{0}=C / 2$ (see also Figs. 8 and 11 for details). 
At finite $N$, the coupling with the low-frequency modes strongly affects the QPS amplitude. Indeed, we find that $v(N) \sim v_{0} / N^{\alpha}$ for $N \gg 1$ where $\alpha \propto\left(E_{J} / E_{0}\right)^{1 / 2}$. Depending on the value of $\alpha, v_{\text {ring }}=N v(N) \sim N^{1-\alpha}$ either tends to zero, when $\alpha \gg 1$, or grows linearly, when $\alpha \ll 1$, indicating that the system either displays a superconducting or an insulating behavior, as can be seen in Fig. 2(b). This behavior is reminiscent of the dissipative phase transition ${ }^{33}$ occurring in a single junction in an electromagnetic environment. ${ }^{14,15}$ For intermediate ring sizes, finite-size effects occur, yielding a nonmonotonic behavior of the maximum supercurrent in the insulating regime, similar to what we find for the case $C_{0}=0$.

The paper is structured as follows. In Sec. III, we recall the model for a flux-biased $1 \mathrm{D} \mathrm{JJ}$ ring as well as the notion of QPS and the approximations involved to find the ring's flux-dependent quantum ground state and hence the maximum supercurrent. In Sec. IV, we discuss the single QPS approximation and we show how the system reduces to a model similar to that of Caldeira and Leggett ${ }^{32}$ where one single Josephson junction, the center of the QPS, is coupled to $N-1$ harmonic oscillators. The results for the specific case $C_{0}=0$ are shown in Sec. V, where we explain in detail the different finite-size corrections on the QPS amplitude. The effect of the finite ground capacitance $C_{0}>0$ and the general results are discussed in Sec. VI. In Sec. VII, we draw our conclusions.

\section{MODEL}

\section{A. Hamiltonian}

We consider a homogeneous ring of $N$ identical superconducting islands each coupled to its nearest neighbor by Josephson tunnel junctions (see Fig. 1). The ring is threaded by a magnetic flux $\Phi_{B}$. We assume the superconducting gap $\Delta$ of the islands to be the largest energy scale involved in the problem. If $\Delta \gg \delta E$ where $\delta E$ is the average spacing of the electronic energy levels, superconductivity is well established. The islands should be metallic with large enough volume so that the perturbative treatment of Cooper-pair tunneling through the contacting surfaces is justified. We assume the absence of quasiparticle excitations at low temperature $T$ and low voltage $k_{B} T, 2 e \bar{V} \ll \Delta$, where $\bar{V}$ is the typical voltage across the junctions. Furthermore, the condition $\Delta \gg E_{C}, E_{0}$ implies that the Josephson coupling energy $E_{J}$ characterizing Cooperpair tunneling between islands is independent of $E_{C}, E_{0}$.

We assume that the kinetic inductance, associated with the kinetic energy of the Cooper pairs in each superconducting island, is negligible as compared to the Josephson inductance..$^{34,35}$ We also assume that the geometric inductance can be neglected so that the current circulating in the ring does not generate any magnetic field. The total flux $\Phi_{B}$ is thus only given by the externally applied magnetic field.

The previous conditions define the standard quantum-phase model for a 1D JJ homogeneous chain whose Hamiltonian reads as ${ }^{16}$

$$
\begin{aligned}
H= & \frac{1}{2} \sum_{n, m=0}^{N-1} \hat{Q}_{n} \overline{\bar{C}}_{n m}^{-1} \hat{Q}_{m} \\
& -E_{J} \sum_{n=0}^{N-1} \cos \left(\hat{\varphi}_{n+1}-\hat{\varphi}_{n}+\frac{2 \pi \Phi_{B}}{N \Phi_{0}}\right),
\end{aligned}
$$

where $\Phi_{0}$ is the flux quantum. For each island, the BCS condensate phase $\hat{\varphi}_{n}$ and the excess charge $\hat{Q}_{n}$ on the $n$th island represent the conjugate variables of the system $\left[\hat{\varphi}_{n}, \hat{Q}_{n}\right]=2 e i$. $\overline{\bar{C}}$ is the capacitance matrix with matrix elements $\overline{\bar{C}}_{n, m}=$ $\left(C_{0}+2 C\right) \delta_{n, m}-C\left(\delta_{n+1, m}+\delta_{n-1, m}\right)$, with the index $n=-1$ corresponding to $N-1$ and $n=0$ corresponding to $N$. The relative phase difference across the $n$th junction is $\hat{\theta}_{n}=\hat{\varphi}_{n+1}-$ $\hat{\varphi}_{n}$. As the phases are compact variables, i.e., $\hat{\varphi}_{N}=\hat{\varphi}_{0}+2 \pi m$ where $m$ is an integer, we have the constraint on the phase differences for Josephson junctions in a ring ${ }^{36}$

$$
\sum_{n=0}^{N-1} \hat{\theta}_{n}=2 \pi m .
$$

Note that the argument of each cosine in Eq. (2) is the gauge-invariant phase difference across the corresponding junction.

From Eqs. (2) and (3), we see that the physical properties of the system depend periodically on the ratio $\delta=2 \pi \Phi_{B} / \Phi_{0}$. In the steady-state regime, the dc supercurrent flowing through the ring is the same for all the junctions $\left\langle\hat{I}_{n}\right\rangle=I$ and can be related to the derivative of the ground-state energy $E_{\mathrm{GS}}$ of the system with respect to $\delta$ :

$$
I(\delta)=\frac{\partial E_{\mathrm{GS}}}{\partial \Phi_{B}}=\left(\frac{2 e}{\hbar}\right) \frac{\partial E_{\mathrm{GS}}}{\partial \delta} .
$$

It is in general a difficult task to find the ground-state energy $E_{\mathrm{GS}}(\delta)$ for the flux-biased ring described by Hamiltonians (2) and (3). An approximate solution can be found in the limit where the Josephson energy is larger than the characteristic electrostatic energy $E_{J} \gg E_{C}, E_{0}$, which is the regime discussed in this paper.

\section{B. Single QPS in JJ rings of finite circumference}

To set the stage, let us first consider the classical limit, achieved by setting $E_{C}=E_{0}=0$, so that the phases are welldefined classical variables. The classical energy of the system reduces to

$$
E_{\mathrm{cl}}=-E_{J} \sum_{n=0}^{N-1} \cos \left(\theta_{n}+\frac{\delta}{N}\right) .
$$

The energy Eq. (5) is invariant under a change by $2 \pi$ of the phases $\theta_{n}$. In other words, the states $\theta_{n}$ and $\theta_{n}+2 \pi k$ are equivalent ( $k$ integer). However, at fixed magnetic flux $\delta$, a given configuration of $\left\{\theta_{n}\right\}$ corresponds to a real physical state only if the constraint Eq. (3) is satisfied. Therefore, any distribution of the phases that violates Eq. (3) is unphysical.

The classical states $|m\rangle$ that minimize the energy Eq. (5) under the constraint (3) correspond to a uniform distribution of phase differences $\theta_{n}=2 \pi m / N$. They have energies

$$
E_{m}=-E_{J} N \cos \left(\frac{2 \pi m+\delta}{N}\right),
$$

with the condition $d^{2} E_{\mathrm{cl}} /\left.d \theta_{n}^{2}\right|_{m}=-E_{m}>0$ and the index $-(N-1) / 2<m<(N-1) / 2$ ( $N$ odd) or $-N / 2<m<$ $(N / 2)-1(N$ even). These classical states $|m\rangle$ are physically distinguishable as they are characterized by different persistent 
currents

$$
I_{m}=I_{J} \sin \left(\frac{2 \pi m+\delta}{N}\right) .
$$

Away from the degeneracy points $\delta=0$ and $\pi$ they also have different energies. The classical ground state corresponds to an absolute minimum

$$
\begin{aligned}
E_{\mathrm{GS}}^{(\mathrm{cl})} & =-E_{J} N \max _{m} \cos \left(\frac{2 \pi m+\delta}{N}\right) \\
& \simeq \frac{E_{J}}{2 N} \min _{m}(2 \pi m+\delta)^{2},
\end{aligned}
$$

where the second, approximate equality is numerically accurate for sufficiently long rings $(N \gtrsim 5)$. The corresponding supercurrent then has as a sawtoothlike dependence as a function of $\delta$ with a maximum supercurrent given by $I_{\mathrm{cl}} \simeq$ $\pi I_{J} / N .^{24}$

For finite $C, C_{0}$, the electrostatic interaction acts as an inertial term on the phases so that quantum fluctuations occur, giving rise to quantum phase slips (QPSs). At fixed magnetic flux and in a ring of finite circumference, the QPS is a collective process corresponding to the quantum tunneling in a multidimensional space of dimension $N$ between two distinct minima of the potential, corresponding for instance to the classical states $|m\rangle$ and $|m+1\rangle$, separated by some energy barrier associated with the potential (5). In the multidimensional space, the physical paths $\left\{\theta_{n}\right\}$ which connect $|m\rangle$ and $|m \pm 1\rangle$ correspond to a subspace defined by the constraint (3). Due to this constraint, the multidimensional tunneling reduces to one-dimensional tunneling in which we have only a few trajectories connecting the initial and the final states (see Fig. 3). As it was discussed in Ref. 24, an example of QPS connecting the states $|m\rangle$ and $|m+1\rangle$ is given by the displacements

$$
\begin{aligned}
\theta_{n_{0}} & =\frac{2 \pi m}{N} \longrightarrow \frac{2 \pi(m+1)}{N}-2 \pi, \\
\theta_{n} & =\frac{2 \pi m}{N} \longrightarrow \frac{2 \pi(m+1)}{N} \quad\left(n \neq n_{0}\right),
\end{aligned}
$$

in which the local phase difference $\theta_{n_{0}}, n_{0}$ being the center of the QPS, winds by an amount of $2 \pi(1+1 / N)$ and the whole set of phase differences $\left\{\theta_{n}\right\}\left(n \neq n_{0}\right)$ shifts in order to preserve the constraint. Figure 3(a) shows this process. One can express the classical energy Eq. (5) as $E_{\mathrm{cl}} / E_{J}=-\cos \left(\theta_{n_{0}}+\delta / N\right)-$ $(N-1) \cos \left(\theta_{n}+\delta / N\right)$ with $n \neq n_{0}$ [Fig. 3(b)].

As it is shown in Fig. 3(b), the trajectory of the QPS equations (9) and (10) can be drawn in a restricted, compact zone scheme as well as in an extended one. This fact allows us to introduce the adiabatic potential for the QPS process. In the limit in which the evolution of the phases is extremely slow, the kinetic energy can be neglected at any time and one obtains the energy of the system just minimizing the classical energy for each intermediate configuration which connects the initial and the final states. Hence, the adiabatic potential is associated with the line of minimum energy on the surface (5) which connects the end points as shown in the example of Fig. 3(b). This line is given by the condition

$$
\theta_{n}=\frac{2 \pi m-\theta_{n_{0}}}{N-1}\left(n \neq n_{0}\right)
$$

(a)
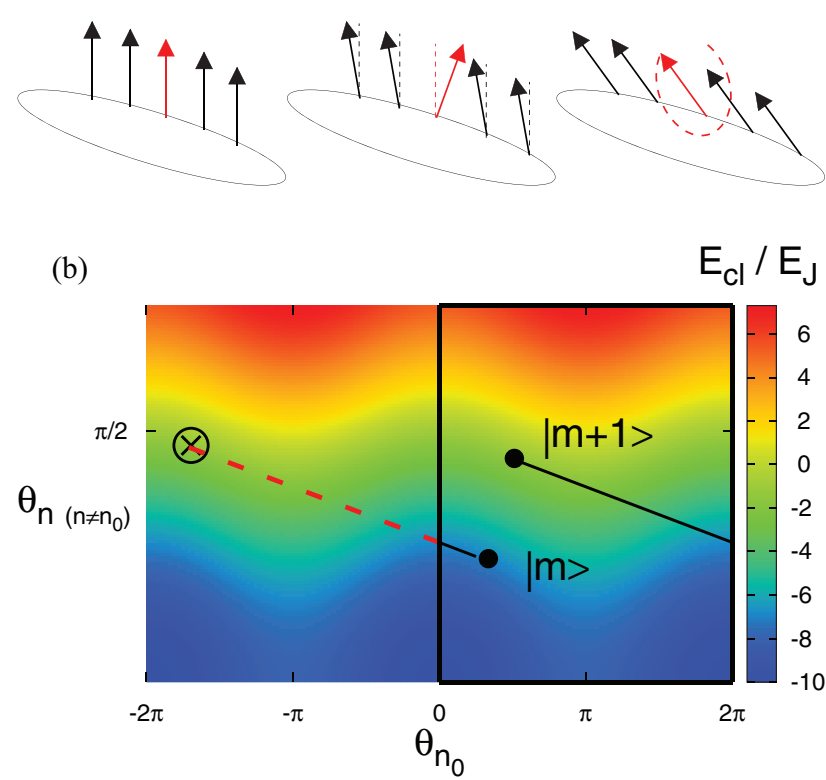

FIG. 3. (Color online) Example of a QPS process. (a) The oval loop represents the JJ ring. The arrows represent a few phase differences $\left\{\theta_{n}\right\}$ around the QPS center at $n_{0}$, the phase $\theta_{n_{0}}$ winding of $2 \pi(1-1 / N)$ (red arrow). The initial configuration (left) is the state $|m\rangle$ and the final configuration (right) is $|m+1\rangle$. The central configuration is intermediate between the two states $|m\rangle$ and $|m+1\rangle$. (b) The classical energy expressed as $E_{\mathrm{cl}} / E_{J}=-\cos (x)-(N-1)$ $\cos (y)$ with the axis $x=\theta_{n_{0}}-\delta / N$ and $y=\theta_{n}-\delta / N\left(n \neq n_{0}\right)$. The dots represent the states $|m\rangle$ and $|m+1\rangle$. The cross also represents the state $|m+1\rangle$ but in the extended zone scheme. The black lines represent the physical trajectory of the QPS that connects the initial and the final states, the (black) solid one in the restricted zone scheme, the (red) dashed line in the extended zone scheme. The bold line for the borders marks the compact region. Parameters: $N=10, \delta=\pi / 2, m=1$.

After a shift of the phase $\theta_{n_{0}} \rightarrow \theta_{n_{0}}-\delta / N$, the effective adiabatic potential $V_{\text {eff }}\left(\theta_{n_{0}}\right)$ reads as

$$
\begin{aligned}
& V_{\mathrm{eff}}\left(\theta_{n_{0}}\right) \\
& \quad=-E_{J}\left[\cos \left(\theta_{n_{0}}\right)+(N-1) \cos \left(\frac{\delta+2 \pi m-\theta_{n_{0}}}{N-1}\right)\right] \\
& \simeq E_{J}\left[-\cos \left(\theta_{n_{0}}\right)+\frac{\left(\delta+2 \pi m-\theta_{n_{0}}\right)^{2}}{2(N-1)}\right]
\end{aligned}
$$

where the second, approximate equality is valid for sufficiently long rings $(N \gtrsim 5)$. At the initial time, we have $V_{\text {eff }}\left[\theta_{n_{0}}=\right.$ $(\delta+2 \pi m) / N]=E_{m}$ and at the final time $V_{\text {eff }}\left\{\theta_{n_{0}}=2 \pi+\right.$ $[\delta+2 \pi(m+1)] / N\}=E_{m+1}$. In Fig. 4, we show $V_{\text {eff }}\left(\theta_{n_{0}}\right)$.

In the limit of $N \rightarrow \infty$, we have $V_{\text {eff }}\left(\theta_{n_{0}}\right)=-E_{J} \cos \left(\theta_{n_{0}}\right)$ and we recover the simplified picture of QPS corresponding to the quantum tunneling of the phase difference $\theta_{n_{0}}$ of a given junction $n_{0}$ from one minimum of the local Josephson potential $-\cos \left(\theta_{n_{0}}\right)$ to the neighboring one.

On the other hand, as we explained above, QPS is a collective process corresponding to a quantum tunneling in a multidimensional space with the constraint (3). Consequently, the potential in terms of the variable $\theta_{n_{0}}$, the center of the QPS, 


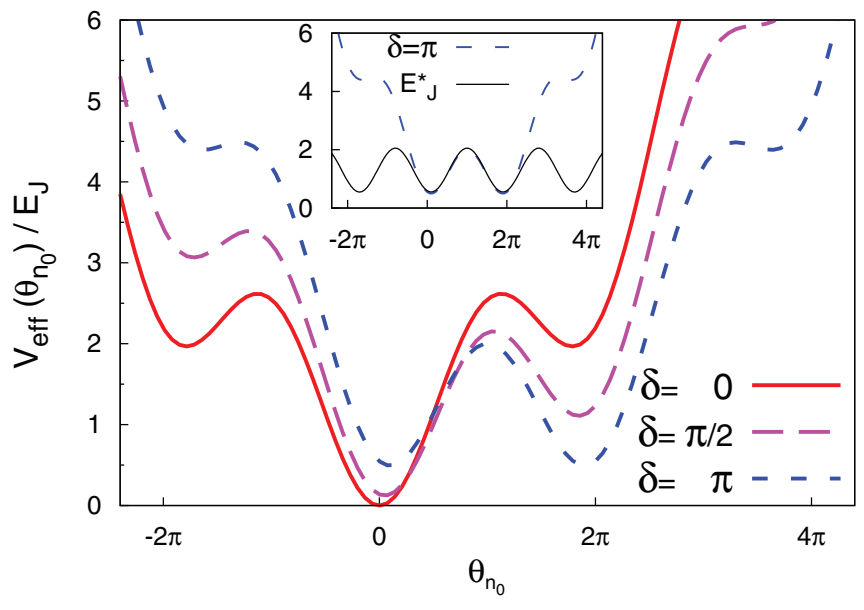

FIG. 4. (Color online) Example of the effective potential $V_{\text {eff }}\left(\theta_{n_{0}}\right)$ [Eq. (12)] for $N=10$ and for the values $\delta=0$ solid (red) line, $\delta=\pi / 2$ dashed (purple) line, and $\delta=\pi$ dotted (blue) line. Inset: comparison between the effective potential at $\delta=\pi$ [dotted (blue) line], with the cosine potential $E_{J}^{*} \cos \left[\theta_{n_{0}}(N-1) / N\right.$ ] [solid line (see text)].

is not a periodic function although the global classical energy of the system Eq. (5) is $2 \pi$ periodically invariant.

\section{Effective low-energy description}

We now turn to the effects of quantum phase fluctuations in the limit $E_{J} \gg E_{C}, E_{0}$. In this case, a simple analysis is possible since the QPS processes occur only rarely, with an exponentially small amplitude $v$ [Eq. (1)]. The single QPS approximation is further analyzed in Secs. VID and VIE, where we estimate its range of validity.

As a consequence of QPSs and for small amplitude $v$, the quantum ground state $\left|\Psi_{\mathrm{GS}}\right\rangle$ of the ring corresponds to a superposition of different classical states of the phases, namely, $\left|\Psi_{\mathrm{GS}}\right\rangle=\sum_{m} c_{m}|m\rangle$. The coefficients $c_{m}$ as well as the quantum ground-state energy $E_{\mathrm{GS}}(\delta)$ can be obtained from the following effective Schrödinger equation: ${ }^{24}$

$$
E_{m} c_{m}-v_{\text {ring }}\left(c_{m+1}+c_{m-1}\right)=E_{\mathrm{GS}} c_{m},
$$

where the term proportional to $v_{\text {ring }} \equiv N v$ connects two classical states differing by a single QPS. The factor $N$ takes into account the fact that a QPS can have the center in any junction of the chain and this corresponds to different trajectories in the multidimensional space $N$ so that QPS amplitudes add up coherently. This coherence has been recently confirmed experimentally in a short 6-SQUID JJ chain ${ }^{37}$ by the measurements of the Aharonov-Casher interference effect. The coherence is affected by offset charge dynamics. The details of this dynamics are currently not understood. It is expected to give rise to an additional dependence of $v$ on $N$ which is beyond the scope of this paper.

The behavior of the general solution for the ground state of the model given by Eq. (13) is determined by only one dimensionless parameter

$$
q=\frac{N^{2} v(N)}{2 \pi^{2} E_{J}} .
$$

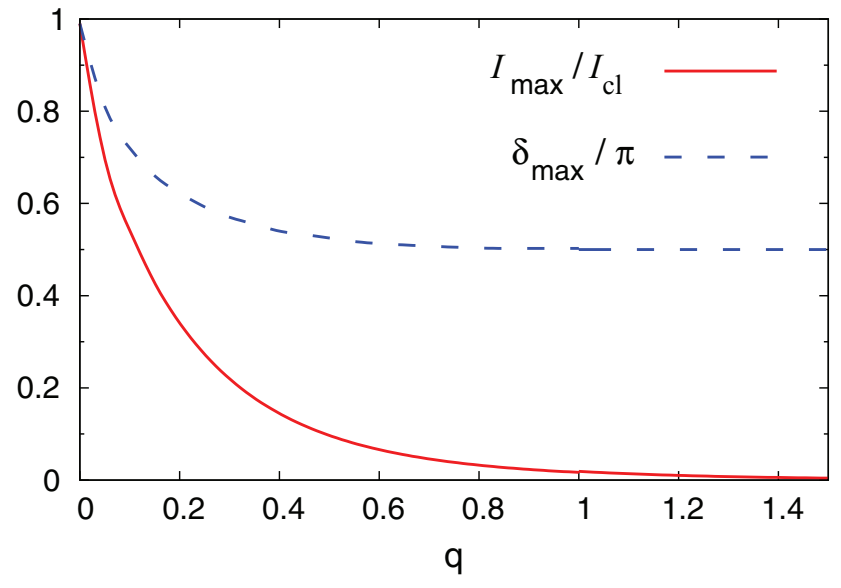

FIG. 5. (Color online) Maximum supercurrent (solid line) for the model defined by Eq. (13) as a function of $q$ [Eq. (14)]. The dashed line represents the value of the scaled flux $\delta_{\max }$ which gives the maximum supercurrent.

The ground-state energy $E_{\mathrm{GS}}=E_{\mathrm{GS}}(\delta ; q)$ depends parametrically on $q$, hence leading to a dependence of the maximum supercurrent $I_{\max }(q)$ on the QPS amplitude $v$. This dependence is illustrated in Fig. 5 together with the evolution of the value of the phase $\delta_{\max }$ that corresponds to the maximum. For $q \ll 1$, the solution scales as $I_{\max } / I_{\mathrm{cl}} \simeq 1-(5 / 2) q^{2 / 3}$, whereas it scales as $I_{\max } / I_{\mathrm{cl}} \simeq 24 \sqrt{\pi} q^{4 / 3} \exp (-8 \sqrt{q})$ for $q \gg 1$.

The effective low-energy theory defined by (13) was introduced in Ref. 24 for $C_{0}=0$ and was discussed for long $\mathrm{JJ}$ rings $N \gg 1$. As we discussed in Sec. III B, the validity of Eq. (13) extends more generally to include rings of intermediate size and with $C_{0} \neq 0$. In the following, we will obtain the detailed dependence of the QPS amplitude $v_{\text {ring }}$ on the parameters $N, E_{J}, E_{C}$, and $E_{0}$. Hence, we calculate the ground-state energy of the system using Eq. (13) to obtain the periodic dependence of $E_{\mathrm{GS}}(\delta)$ and $I(\delta)$ on $\delta$ [Eq. (4)] from which we extract $I_{\max }$.

\section{QPS IN 1D JJ RING}

\section{A. General approach for a single QPS event}

We present a general approach to calculate the quantum amplitude $v$ for a single QPS event occurring on a ring containing $N$ junctions and with mutual and ground capacitance $C$ and $C_{0}$, in the regime $E_{J} \gg E_{C}, E_{0}$. According to Eqs. (9) and (10), the QPS process is a collective process in which the local phase difference $\theta_{n_{0}}$ winds by an amount of $2 \pi(1-1 / N)$, accompanied by a shift of the whole set of phase differences $\left\{\theta_{n}\right\}\left(n \neq n_{0}\right)$. For rings of circumference $N \gtrsim 5$, the phase differences of the other junctions will vary only slightly $\Delta \theta_{n} \sim 1 / N$ compared to the period $2 \pi$ of the cosine potential. Hence, we can apply the harmonic approximation to describe the dynamics corresponding of $\theta_{n}, n \neq n_{0}$.

For vanishing ground capacitance $C_{0}=0$, the phase differences of the other $N-1$ junctions behave as independent LC oscillators at the plasma frequency $\omega_{p}$ whose displacement is inversely proportional to the circumference $N$. Thus, in the limit of a very large ring $N \gg 1$, the dynamics of the other $N-1$ phase differences can be neglected ${ }^{24}$ so that the QPS 
amplitude $v(N)$ can be approximated by the $N$-independent constant value $v_{0}$ [Eq. (1)]. However, for $1 \mathrm{D} \mathrm{JJ}$ rings of finite circumference, the dynamics of the other junctions can have considerable effects on $v$, as we will show in the following. Moreover, for finite ground capacitance $C_{0}>0$, the harmonic oscillations of the phase differences of the $N-1$ junctions play a crucial role for any ring's circumference.

To calculate the QPS amplitude $v$, we start by considering the partition function associated with the Hamiltonian (2), with the constraint (3). In the path-integral formalism, it reads as $\left(\beta=\hbar / k_{B} T\right)$

$$
\mathcal{Z}=\operatorname{Tr}\left[e^{-\frac{\beta}{\hbar} \hat{H}}\right]=\prod_{n=0}^{N-1} \oint \mathcal{D}\left[\varphi_{n}(\tau)\right] e^{-\mathcal{S} / \hbar},
$$

where the Euclidean action for the phases $\left\{\varphi_{n}(\tau)\right\}$ reads as $\mathcal{S}=\int_{0}^{\beta} d \tau \mathcal{L}$, and $\mathcal{L}$ is the Lagrangian

$$
\begin{aligned}
\mathcal{L}= & \sum_{n=0}^{N-1} \frac{\hbar^{2} C_{0}}{8 e^{2}} \dot{\varphi}_{n}^{2}+\sum_{n=0}^{N-1} \frac{\hbar^{2} C}{8 e^{2}}\left(\dot{\varphi}_{n+1}-\dot{\varphi}_{n}\right)^{2} \\
& -\sum_{n=0}^{N-1} E_{J} \cos \left(\varphi_{n+1}-\varphi_{n}+\frac{\delta_{m}}{N}\right),
\end{aligned}
$$

with $\dot{\varphi}_{n}=d \varphi / d \tau$ and $\delta_{m}=\delta+2 \pi m$. The compact variables $\left\{\varphi_{n}\right\}$ are defined on the circle $[0 ; 2 \pi$ [. Notice that we shifted the phase differences $\left\{\theta_{n}\right\}$ with respect to their average value so that Eq. (3) now reads as $\sum_{n=0}^{N-1} \theta_{n}=0$. The last constraint is automatically satisfied by imposing the boundary condition $\varphi_{N}=\varphi_{0}$.

\section{B. Harmonic modes}

Let us briefly discuss the behavior of the system in the harmonic approximation, neglecting the QPS. When the Josephson energy $E_{J} \gg E_{C}, E_{0}$ the phases fluctuate only slightly around their classical values. The average phase difference between the neighboring islands is small so that we can expand the Josephson interaction to lowest (quadratic) order. The general imaginary-time Lagrangian (16) then reduces to the harmonic one

$$
\begin{aligned}
\mathcal{L}_{\mathrm{har}}^{(N)}= & \sum_{n=0}^{N-1} \frac{\hbar^{2} C_{0}}{8 e^{2}} \dot{\varphi}_{n}^{2}+\sum_{n=0}^{N-1} \frac{\hbar^{2} C}{8 e^{2}}\left(\dot{\varphi}_{n+1}-\dot{\varphi}_{n}\right)^{2} \\
& +\sum_{n=0}^{N-1} \frac{1}{2} E_{J}\left(\varphi_{n+1}-\varphi_{n}\right)^{2}+\frac{E_{J}}{2 N} \delta_{m}^{2}
\end{aligned}
$$

where we omitted an irrelevant constant term. Any periodic function $\varphi_{n}$ defined on the finite lattice $n=0, \ldots, N-1$ can be decomposed as

$$
\varphi_{n}=\frac{1}{\sqrt{N}} \sum_{k=0}^{N-1} \varphi_{k} e^{i \frac{2 \pi}{N} k n}
$$

with the condition for the complex variables $\varphi_{N-k}=\varphi_{k}^{*}$ which preserves the total number of degrees of freedom. Substituting Eq. (18) into (17) and summing over the index $n$, the harmonic

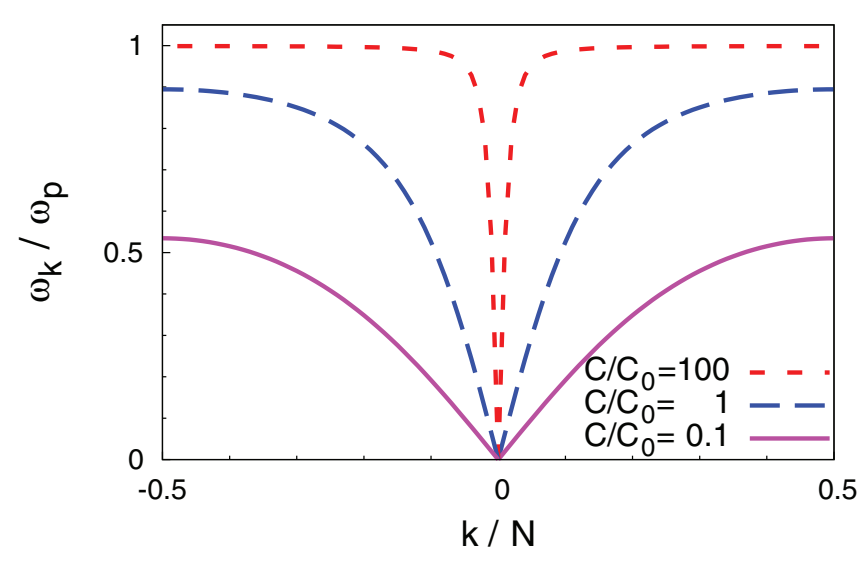

FIG. 6. (Color online) Frequency dispersion of the harmonic modes in a $\mathrm{JJ}$ ring of $N=100$ junctions. We use the equivalent index notation $k=-N / 2+1, \ldots, N / 2$. Solid (purple) line, dashed (blue) line, and dotted (red) line are, respectively, for the ratio $C / C_{0}=0.1,1,100$.

Lagrangian is diagonalized (see also Appendix A):

$$
\mathcal{L}_{\text {har }}^{(N)}=\sum_{k=0}^{N-1}\left(\frac{1}{2} \mu_{k}\left|\dot{\varphi}_{k}\right|^{2}+\frac{1}{2} \mu_{k} \omega_{k}^{2}\left|\varphi_{k}\right|^{2}\right)+\frac{E_{J}}{2 N} \delta_{m}^{2} .
$$

We have introduced the constants

$$
\mu_{k}=\frac{\hbar^{2}}{4 e^{2}}\left\{C_{0}+2 C\left[1-\cos \left(\frac{2 \pi}{N} k\right)\right]\right\} ;
$$

the frequency dispersion is given by

$$
\omega_{k}=\omega_{p} \sqrt{\frac{1-\cos \left(\frac{2 \pi k}{N}\right)}{1-\cos \left(\frac{2 \pi k}{N}\right)+\frac{\pi^{2}}{2 \lambda^{2}}}},
$$

where the screening length is $\lambda=\pi \sqrt{C / C_{0}}$. For $N \gg 1$, the maximal frequency of the modes $\omega_{\max }$ is given by

$$
\omega_{\max }=4 \sqrt{\frac{E_{J}}{\hbar^{2}}\left(\frac{e^{2}}{4 C+C_{0}}\right)} .
$$

An example of this frequency dispersion is given in Fig. 6. We can distinguish two regimes for $C_{0}>0$. For $\mathrm{JJ}$ rings longer than the screening length $\lambda, N \gg \lambda$, the spectrum has a linear dispersion for low frequencies. For shorter rings $N \ll \lambda$, the lowest mode appears almost at the plasma frequency $\omega_{p}$ and the linear behavior is completely absent. For the case $C_{0}=0$, we recover a flat distribution where all the modes are degenerate and correspond to the plasma frequency $\omega_{p}$.

Let us calculate the phase-dependent ground-state energy $E_{\mathrm{GS}}^{(\mathrm{har})}(\delta)$ in the harmonic approximation. The path integral in Eq. (15) can be explicitly calculated for the diagonalized harmonic action (19). We obtain

$$
\mathcal{Z}_{\mathrm{har}}^{(N)} \sim\left(\prod_{k \neq 0} \frac{1}{2 \sinh \left(\beta \omega_{k} / 2\right)}\right) e^{-\frac{\beta E_{J}}{2 \hbar N} \min _{m} \delta_{m}^{2}},
$$

where the product corresponds to the partition function for an ensemble of $N-1$ independent harmonic oscillators and the exponential factor contains the classical energy of the 
system $(\bmod 2 \pi)$ at finite temperature. Notice that the zerofrequency mode $k=0$ is not involved in the relevant part of the partition function. Note also the periodicity of the result (23) with respect to $\delta$. The quadratic dependence on $\delta$ in each of the segment $-\pi+2 \pi m<\delta<-\pi+2 \pi(m+1)$ is a consequence of the harmonic approximation, i.e., Eq. (8).

We thus obtain the phase-dependent ground-state energy $E_{\mathrm{GS}}^{(\mathrm{har})}=-\lim _{\beta=\infty}(\hbar / \beta) \ln \mathcal{Z}_{\mathrm{har}}^{(N)}$ :

$$
E_{\mathrm{GS}}^{(\mathrm{har})}=\sum_{k \neq 0} \frac{\hbar \omega_{k}}{2}+\frac{E_{J}}{2 N} \min _{m} \delta_{m}^{2} .
$$

Notice that to reach the full quantum regime of the harmonic modes, as expressed by Eq. (24), the temperature has to be much smaller than the energy of the lowest mode $k_{B} T \ll \hbar \omega_{\min }$, which is given by

$$
\omega_{\min }=\omega_{\max } \frac{\pi}{N} \sqrt{\frac{1+\left(\frac{2 \lambda}{\pi}\right)^{2}}{1+\left(\frac{2 \lambda}{N}\right)^{2}}} .
$$

We conclude that, in the absence of QPS processes, the JJ ring forms a closed quantum LC line (each Josephson junction in Fig. 1 is replaced by an inductance $L_{J}=\hbar^{2} / 4 e^{2} E_{J}$ ) formed by $N-1$ independent harmonic oscillators with eigenfrequencies $\omega_{k}$. The classical sawtooth relation between the supercurrent and the total phase $I=I(\delta)$ is unmodified by the quantum harmonic fluctuations of the phases. However, harmonic fluctuations are relevant when the QPSs are restored, as we will now show.

\section{Effective QPS action in the presence of harmonic modes}

We now turn our attention to the effect of the harmonic modes on the QPS amplitude. We will restrict our analysis to the limit in which the frequency of the lowest mode $\omega_{\min }$ is greater than the frequency $v / \hbar$ associated to the tunneling in the effective (static) potential $V_{\text {eff }}$ [Eq. (12)]. In this adiabatic limit, the problem can be reduced to an effective two-state problem involving tunneling between neighboring states $m$ and $m+1$ (see also Sec. VIE).

As discussed in Sec. III B, when a single QPS is centered on one specific junction $n_{0}$, the dynamics of the other junctions $\left(n \neq n_{0}\right)$ is well described by the harmonic approximation. Then, as seen in Sec. IV B, we can consider this part of the ring as an electromagnetic environment formed by $N-1$ independent harmonic oscillators and to which the single junction, center of the QPS process, is coupled. Accordingly, it is natural to cast the full action in a form where the winding phase difference $\theta_{n_{0}}$ is linearly coupled to an ensemble of harmonic oscillators acting as an external bath.

Without loss of generality, one can assume the center of the QPS to the junction $n_{0}=N-1$, namely, $\theta=\theta_{N-1}=$ $\varphi_{0}-\varphi_{N-1}$. It is useful to introduce the average value of the phase at the junction $N-1$, namely, $\Theta_{0}=\left(\varphi_{0}+\varphi_{N-1}\right) / 2$, so that one can write

$$
\varphi_{0}=\Theta_{0}+\frac{\theta}{2}, \quad \varphi_{N-1}=\Theta_{0}-\frac{\theta}{2} .
$$

We choose as the relevant variables the set $S$ given by the winding phase difference difference $\theta$ together with the local phases $\varphi_{n}$ with $n=1, \ldots, N-2$.
First, we discuss the harmonic expansion of the potential energy of the Lagrangian (16). In a QPS, the phase differences across the junctions remain small with respect to $2 \pi$ except at the junction $n_{0}=N-1$ :

$$
\begin{aligned}
\sum_{n=0}^{N-1} \cos \left(\theta_{n}+\frac{\delta_{m}}{N}\right) \simeq & \cos \left(\theta+\frac{\delta_{m}}{N}\right) \\
& -\frac{1}{2} \sum_{n=0}^{N-2}\left(\theta_{n}+\frac{\delta_{m}}{N}\right)^{2} .
\end{aligned}
$$

Using the set $S$, the last sum in Eq. (27) contains a quadratic coupling between $\theta$ and the quantities $\Theta_{0}-\varphi_{1}$ and $\Theta_{0}-\varphi_{N-2}$. Although these terms contain $\theta$ varying by almost $2 \pi$, this does not make invalid our expansion as the overall argument of the cosine function, representing the phase difference in the neighboring junctions $n=0$ and $N-2$ remains small during the QPS.

Using the set $S$, the Lagrangian of Eq. (16) is decomposed as

$$
\mathcal{L}=\mathcal{L}_{1}+\mathcal{L}_{2}+\mathcal{L}_{3},
$$

where the first term $\mathcal{L}_{1}$ is associated with the winding junction

$$
\begin{aligned}
\mathcal{L}_{1}= & \frac{\hbar^{2}\left(3 C+C_{0}\right)}{16 e^{2}} \dot{\theta}^{2}-E_{J} \cos \left(\theta+\frac{\delta_{m}}{N}\right) \\
& +E_{J}\left(\frac{\theta^{2}}{4}-\theta \frac{\delta_{m}}{N}\right) .
\end{aligned}
$$

The second term $\mathcal{L}_{2}$ describes the environment to which $\theta$ is coupled. Hereafter, we change the notation for the average

$$
\Theta_{0} \rightarrow \varphi_{0}
$$

in order to simplify the following formulas. Then, $\mathcal{L}_{2}$ reads as

$$
\begin{aligned}
\mathcal{L}_{2}= & \frac{\hbar^{2} C_{0}}{8 e^{2}} \dot{\varphi}_{0}^{2}+\sum_{n=0}^{N-2} \frac{\hbar^{2}}{8 e^{2}}\left[C_{0} \dot{\varphi}_{n}^{2}+C\left(\dot{\varphi}_{n+1}-\dot{\varphi}_{n}\right)^{2}\right] \\
& +\sum_{n=0}^{N-2} \frac{1}{2} E_{J}\left(\varphi_{n+1}-\varphi_{n}\right)^{2}+E_{J}(N-1)\left(\frac{\delta_{m}}{N}\right)^{2},
\end{aligned}
$$

with the periodic boundary condition $n=0$ corresponding to $n=N-1$. Note the extra term in Eq. (31) associated with the (average) phase at $n=0$. The last term $\mathcal{L}_{3}$ of Eq. (28) describes the coupling between the winding junction and the electromagnetic environment,

$$
\mathcal{L}_{3}=\frac{\hbar^{2} C}{8 e^{2}}\left(\dot{\varphi}_{N-2}-\dot{\varphi}_{1}\right) \dot{\theta}+\frac{E_{J}}{2}\left(\varphi_{N-2}-\varphi_{1}\right) \theta .
$$

We rewrite the Lagrangians $\mathcal{L}_{2}$ and $\mathcal{L}_{3}$ in terms of the normal modes $\varphi_{k}$. They are given by Eq. (18) with $N$ replaced by $N-1$. In terms of these modes, we have

$$
\mathcal{L}_{2}=\frac{\hbar^{2} C_{0}}{8 e^{2}} \dot{\varphi}_{n=0}^{2}+\mathcal{L}_{\mathrm{har}}^{(N-1)}+\frac{E_{J}}{2} \delta_{m}^{2}\left(\frac{N-2}{N(N-1)}\right),
$$

where $\mathcal{L}_{\text {har }}^{(N-1)}$ is defined by Eqs. (19), (20), and (21) in which we have to replace $N$ by $N-1$. Then, from Eq. (32), one can see that the phase $\theta$ is coupled only to the imaginary part of 
the modes $\varphi_{k}=\varphi_{k}^{R}+i \varphi_{k}^{I}$ (see Appendix A):

$$
\mathcal{L}_{3}=\sum_{k=1}^{k_{\max }} \zeta_{k}\left(\frac{\hbar^{2} C}{8 e^{2}} \dot{\varphi}_{k}^{I} \dot{\theta}+\frac{E_{J}}{2} \varphi_{k}^{I} \theta\right),
$$

where $k_{\max }=(N-3) / 2$ for $N$ odd and $k_{\max }=(N-2) / 2$ for $N$ even. We have introduced the factor $\zeta_{k}$ :

$$
\zeta_{k}=4 \sin [2 \pi k /(N-1)] /(N-1)^{1 / 2} \text {. }
$$

Notice that the mode $k=0$ is not coupled to the winding phase $\theta$ and we can omit it hereafter. The partition function associated with the total Lagrangians (28), (29), (33), and (34) reads as

$$
\mathcal{Z} \sim \oint \mathcal{D}[\theta(\tau)] \prod_{k=1}^{k_{\max }} \oint \mathcal{D}\left[\varphi_{k}^{I}(\tau)\right] e^{-\frac{1}{\hbar} \int_{0}^{\beta} d \tau\left(\mathcal{L}_{1}+\mathcal{L}_{2}+\mathcal{L}_{3}\right)} .
$$

It is possible to integrate out the imaginary parts of the $N-2$ harmonic modes to obtain a single effective action describing the dynamics of $\theta$. After the calculation, shown in Appendix A, we find

$$
\mathcal{Z} \sim \mathcal{Z}_{\text {har }}^{(N-2)} \oint \mathcal{D}[\theta(\tau)] e^{-S_{\text {eff }}[\theta(\tau)]},
$$

where $\mathcal{Z}_{\text {har }}^{(N-2)}$ is given by Eq. (23) with $N-1$ replacing $N$. After a shift of the phase $\theta \rightarrow \theta-\delta_{m} / N$, the effective action for the phase $\theta$ is given by

$$
\begin{aligned}
S_{\mathrm{eff}}= & \int_{0}^{\beta} d \tau\left[\frac{\hbar^{2}}{8 e^{2}}\left(\frac{N C}{N-1}+\frac{C_{0}}{2}\right) \dot{\theta}^{2}\right. \\
& \left.-E_{J} \cos (\theta)+\frac{E_{J}\left(\delta_{m}-\theta\right)^{2}}{2(N-1)}\right] \\
& +\frac{1}{2} \int_{0}^{\beta} d \tau \int_{0}^{\beta} d \tau^{\prime} G\left(\tau-\tau^{\prime}\right) \theta(\tau) \theta\left(\tau^{\prime}\right) .
\end{aligned}
$$

The effective action has a kernel $G(\tau)$ which is nonlocal in time and whose Fourier series is given by $G(\tau)=$ $\sum_{\ell}(1 / \beta) G_{\ell} \exp \left(i \omega_{\ell} \tau\right)$ where $\omega_{\ell}=2 \pi \ell / \beta$ are bosonic Matsubara frequencies and $G_{\ell}$ reads as

$$
G_{\ell}=\frac{\hbar^{2} C_{0}}{4 e^{2}}\left[\frac{\omega_{\ell}^{2}}{2(N-1)}\right] \sum_{k=1}^{k_{\max }} \frac{1+\cos \left(\frac{2 \pi k}{N-1}\right)}{1-\cos \left(\frac{2 \pi k}{N-1}\right)+\frac{\pi^{2}}{2 \lambda^{2}}\left(\frac{\omega_{\ell}^{2}}{\omega_{\ell}^{2}+\omega_{p}^{2}}\right)} .
$$

The kernel has the relevant property $G_{\ell}=0$ for $\ell=\omega_{\ell}=0$. The last relation is equivalent to $\int_{0}^{\beta} d \tau G\left(\tau-\tau^{\prime}\right)=\int_{0}^{\beta} d \tau^{\prime} G(\tau-$ $\left.\tau^{\prime}\right)=0$. As a consequence, this term is invariant under a shift of the winding phase $\theta \rightarrow \theta+$ const. In other words, upon a proper redefinition of $G(\omega)=\omega^{2} G^{\prime}(\omega)$, this (kinetic) term can be written as $\sim G^{\prime}\left(\tau-\tau^{\prime}\right) \dot{\theta}(\tau) \dot{\theta}\left(\tau^{\prime}\right)$.

We observe that the potential in the first line of $S_{\text {eff }}$ in Eq. (38) corresponds exactly to the adiabatic potential $V_{\text {eff }}(\theta)$ [Eq. (12)] introduced in Sec. III B. This potential breaks formally the periodicity in $\theta$ in the action. This symmetry breaking is a consequence of the fact that the QPS is a quantum tunneling in a multidimensional space with the constraint imposed by the magnetic flux threading the $\mathrm{JJ}$ ring (see discussion in Sec. III B).

In summary, Eqs. (37), (38), and (39) constitute the central result of this paper. They enable us to calculate the size-dependent QPS amplitude $v(N)$ and hence the phase-dependent ground-state energy and the ring's maximum supercurrent $I_{\max }$ in a broad range of values of the parameters $N, E_{J}, E_{C}$, and $E_{0}$, as we will show in detail below. However, we first establish a relation with previous work ${ }^{14,15}$ on infinitely long chains by considering the thermodynamic limit.

\section{Thermodynamic limit and the dissipative dynamics}

The effective action (38) describes the single winding junction coupled to its electromagnetic environment constituted by the other $N-2$ junctions in the harmonic approximation. This action is very similar to the one describing the dissipative dynamics of the single Josephson junction in the framework of the Caldeira-Leggett model. ${ }^{32}$ In this model, an abstract bath formed by an infinite number of harmonic oscillators is phenomenologically introduced as the mechanism of irreversible loss of energy in the Josephson junction.

The external bath discussed here, expressed by the kernel $G(\tau)$ in Eq. (39), physically corresponds to the real harmonic modes sustained by the Josephson junction ring. These discrete modes can be experimentally designed and tested. ${ }^{12}$ As long as the ring has finite size, there are a finite number of discrete modes and no real dissipation occurs. We also note that the interaction between the winding local phase difference at the junction $n_{0}$ and these $N-2$ harmonic modes is characterized by a linear coupling through the positions of the oscillators $\varphi_{k}$ as well as through their velocities $\dot{\varphi}_{k}$ [see Eq. (34)]. As we will show now, the difference between the system described by Eqs. (38) and (39) and the standard Caldeira-Leggett model disappears in the limit $N=\infty$.

Let us consider Eqs. (38) and (39). Taking the limit $N \rightarrow \infty$, the first term on the right-hand side of Eq. (38) reduces to the action for a single capacitively shunted Josephson junction with a capacitance $C+C_{0} / 2$. Equation (39) for the kernel $G_{\ell}$ then takes a simple form by replacing the sum with an integral. Proceeding in this way, we add the kinetic term of the first line of Eq. (38) to $G_{\ell}$ to recover Korshunov's result for the total kernel $\gamma\left(\omega_{\ell}\right)$ of a QPS in a chain of infinite length ${ }^{38}$

$$
\begin{aligned}
\gamma\left(\omega_{\ell}\right) & =\frac{\hbar^{2}}{4 e^{2}} \omega_{\ell}^{2}\left(C+\frac{C_{0}}{2}\right)+G_{\ell} \\
& =E_{J}\left(\frac{\omega_{\ell}^{2}}{\omega_{\max }^{2}}\right)+\frac{\hbar \omega_{\ell}}{4} \sqrt{\frac{E_{J}}{2 E_{0}}} \sqrt{1+\frac{\omega_{\ell}^{2}}{\omega_{\max }^{2}}},
\end{aligned}
$$

where $\omega_{\max }$ is defined in Eq. (22). The effective action now reads as

$$
\begin{aligned}
S_{\mathrm{eff}}= & \int_{0}^{\beta} d \tau \int_{0}^{\beta} d \tau^{\prime} \frac{1}{2} \gamma\left(\tau-\tau^{\prime}\right) \theta(\tau) \theta\left(\tau^{\prime}\right) \\
& -\int_{0}^{\beta} d \tau E_{J} \cos [\theta(\tau)] .
\end{aligned}
$$

First, let us discuss the high- and low-energy regions for the dynamics of the winding phase difference $\theta$ in the cosine Josephson potential. These two regions are separated by the condition that the kinetic energy be respectively larger or smaller than the height of the potential $\sim E_{J}$. To estimate the kinetic energy, we determine the effective capacitance of the junction. This can be achieved by taking the limit $\omega_{\ell} \rightarrow \infty$. Then, the kernel $\gamma\left(\omega_{\ell}\right)$ [Eq. (40)] corresponds simply to a pure 
capacitance

$$
\gamma\left(\omega_{\ell}\right) \simeq \frac{\left(\hbar \omega_{\ell}\right)^{2}}{4 e^{2}}\left[C+\frac{C_{0}}{4}+\sqrt{\frac{C_{0}}{4}\left(C+\frac{C_{0}}{4}\right)}\right] .
$$

The kinetic energy corresponds to the height of the potential barrier when $\gamma\left(\omega_{\ell}\right)=E_{J}$, giving the threshold $\omega_{\ell}=\omega_{\max }$. For $\omega_{\ell}>\omega_{\max }$, the cosine Josephson potential is a small perturbation for the dynamics $\theta$. On the contrary, the range $\omega_{\ell}<\omega_{\max }$ corresponds to the energy region where the winding phase $\theta$ moves well within a potential minimum. In the absence of the interaction with the modes, its dynamics is harmonic.

The threshold $\omega_{\max }$ separates the low- and high-frequency parts of the kernel $\gamma$ [Eq. (40)]. As we have explained, the high-frequency component $\omega_{\ell}>\omega_{\max }$ scales approximately as $\gamma\left(\omega_{\ell}\right) \sim \omega_{\ell}^{2}$ and the chain behaves as a pure capacitance. On the other hand, approaching the zero frequency, $\gamma\left(\omega_{\ell}\right)$ scales linearly. In a Josephson junction with dissipation, this behavior corresponds to the effect of an external resistance $R$ leading to a dimensionless damping parameter $\eta=R_{q} / R$ (Ref. 39) where $R_{q}=\hbar / 4 e^{2}$. In our model, for $N=\infty$, this resistance corresponds to $R=\left(L_{J} / C_{0}\right)^{1 / 2}$, the characteristic low-frequency impedance of the chain related to linear dispersion of the modes.

It is interesting to observe that in the standard CaldeiraLeggett model, the two energy scales (the first one related to the ratio between the height of the potential and the kinetic energy and the second one related to the high-energy cutoff for the dissipation) are generally different as the latter is determined by the specific electromagnetic environment to which the junction is coupled.

Defining the relaxation time $\tau_{r}=R C$, we can calculate the quality factor given by the ratio between the oscillation period and the relaxation time $Q=2 \pi /\left(\omega_{p} \tau_{r}\right)=2 \pi\left(C / C_{0}\right)^{1 / 2}$. In infinite Josephson junction chains, the underdamped regime $Q \gg 1$ corresponds thus to $C \gg C_{0}$.

\section{RESULTS: VANISHING GROUND CAPACITANCE}

We now go beyond the thermodynamic limit and discuss finite-size effects for a ring in the limit $C_{0}=0$. The nonlocal kernel then vanishes, $G_{\ell}=0$, and the effective Lagrangian from Eq. (38) reduces to

$$
\mathcal{L}_{\text {eff }}=\left(\frac{N}{N-1}\right) \frac{\hbar^{2} C}{8 e^{2}} \dot{\theta}^{2}-E_{J} \cos (\theta)+\frac{E_{J}\left(\delta_{m}-\theta\right)^{2}}{2(N-1)} .
$$

This result has a simple interpretation. Let us write the total Lagrangian (28), with $C_{0}=0$ in terms of the phase differences $\theta_{n}$,

$$
\mathcal{L}=\frac{\hbar^{2} C}{8 e^{2}} \dot{\theta}-E_{J} \cos (\theta)+\sum_{n=1}^{N-1}\left(\frac{\hbar^{2} C}{8 e^{2}} \dot{\theta}_{n}+\frac{E_{J}}{2} \theta_{n}^{2}\right) .
$$

We observe that the phases $\left\{\theta_{n}\right\}$ are not coupled explicitly to the tunneling phase $\theta$. But, the total phase difference is fixed $\sum_{n} \theta_{n}+\theta=\delta_{m}$ which enforces an implicit interaction between them. In case of identical phase differences $\theta_{n}$ for all
$N-1$ junctions, we have

$$
\theta_{n}=\frac{\delta_{m}-\theta}{N-1}, \quad \dot{\theta}_{n}=-\frac{\dot{\theta}}{N-1} .
$$

By inserting Eq. (45) in the action (44), we obtain the effective action (43). Clearly, in the limit $N \rightarrow \infty$ finite-size corrections vanish and we recover the simple action for the single JJ.

A finite value for $N$ first of all modifies the kinetic term. The constraint-induced coupling to the other junctions increases the inertial mass $C^{*} / C$ of the single phase performing an almost complete winding, leading to a reduction of the charging energy,

$$
\frac{C^{*}}{C}=\frac{N}{N-1}, \quad \frac{E_{C}^{*}}{E_{C}}=1-\frac{1}{N} .
$$

This causes a reduction of the QPS amplitude.

On the other hand, as we can see from Eq. (43), the action of the phase $\theta$ involves the effective adiabatic potential $V_{\text {eff }}(\theta) / E_{J}=1-\cos \theta+(\delta-\theta)^{2} /(N-1) / 2$ (see Fig. 4), which is not purely sinusoidal and depends on $N$, leading to additional finite-size corrections.

Before discussing these corrections, we note that for short rings, the effective potential can have only one minimum for arbitrary value of $\delta$. This is the case, for instance, when $\delta=0$ for $N<5$. In this regime, the harmonic approximation fails too. Focusing on lengths longer than $N \gtrsim 5$, the potential has always two minima for which the QPS process is well defined.

Due to the periodicity in $\delta_{m}$ and the symmetry in $\left(\delta_{m} \leftrightarrow-\delta_{m}\right)$ for the quantum ground state of the system, we can restrict our discussion to values of $\delta$ between 0 and $\pi(m=0)$. In this case, the relevant tunneling process corresponds to an increase of the variable $\theta$. The two positions corresponding to the two minima of the effective potential are, respectively, $\theta_{l}=\delta / N$ and $\theta_{r}=2 \pi+(\delta-2 \pi) / N$, with the energies $V_{\text {eff }}\left(\theta_{l}\right)$ and $V_{\text {eff }}\left(\theta_{r}\right)$, which correspond to the two classical energies associated with the initial and the final states before and after a QPS, respectively.

\section{A. Symmetric effective potential $(q<1)$}

For small values of the parameter $q$ [Eq. (14)], the hopping term is small [Eq. (13)]. The QPS process couples mainly two neighboring classical states $|m\rangle$ and $|m+1\rangle$, and it is significant when they are degenerate. This occurs for $\delta \simeq \pi$ which corresponds to the point of the maximum of the supercurrent in this regime (Fig. 5).

Therefore, we start by discussing the case $\delta=\pi$ when the effective potential $V_{\text {eff }}(\theta)$ is symmetric around the maximum (Fig. 4). Then, the effective potential is very well approximated by a renormalized cosine potential of the form $-E_{J}^{*} \cos [\theta(N-$ $1) / N]$ (see inset Fig. 4) where $E_{J}^{*}$ is the renormalized Josephson energy (half of the height of the energy barrier separating the two wells)

$\frac{E_{J}^{*}}{E_{J}}=\frac{1}{2}\left[1+\cos \left(\frac{\pi}{N}\right)-\frac{\pi^{2}}{2 N^{2}}(N-1)\right] \simeq 1-\frac{\pi^{2}}{4 N}$.

The ratio $E_{J}^{*} / E_{J}$ is an increasing function of $N$ which converges to one in the limit of $N \gg 1$. Thus, for any finite-size system, this correction corresponds to a decrease of the barrier 


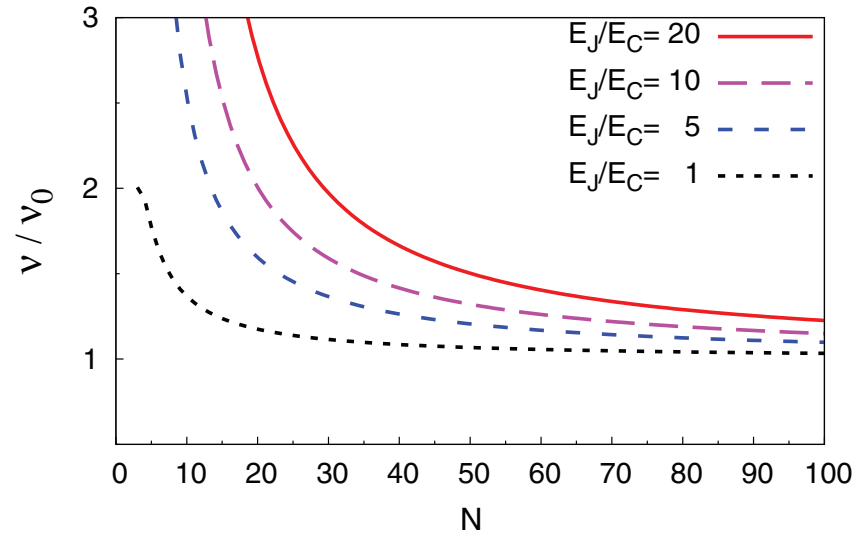

FIG. 7. (Color online) For $C_{0}=0$, the renormalized QPS amplitude $v$ as a function of $N$ [Eq. (49)], scaled with $v_{0}$ [Eq. (1)], for different values of the ratio $E_{J} / E_{C}$.

for quantum tunneling which therefore would lead to an enhancement of the QPS amplitude, in contrast to the effect of the renormalized capacitance [Eq. (46)]. Another effect which enhances the tunneling amplitude is the reduction of the distance between the two minima of the effective potential

$$
\frac{\Delta \theta^{*}}{2 \pi}=1-\frac{1}{N},
$$

contributing to the enhancement of the tunneling amplitude for finite $N$. Similar renormalization effects have been discussed in Ref. 28, albeit for different superconducting circuits.

The three different effects given by Eqs. (46), (47), and (48) combine in the final expression for the renormalized effective amplitude for the quantum tunneling between the two degenerate minima

$$
\nu(N)=\sqrt{\left(\frac{2 \pi}{\Delta \theta^{*}}\right)} \frac{4}{\sqrt{\pi}}\left(8 E_{J}^{* 3} E_{C}^{*}\right)^{\frac{1}{4}} e^{-\left(\frac{\Delta \theta^{*}}{2 \pi}\right) \sqrt{8 \frac{E_{J}^{*}}{E_{C}^{*}}} .}
$$

For the range of interest $N \geqslant 5$, the QPS amplitude (49) decreases with the length $N$ indicating that the latter effects (reduced barrier height and distance between minima) dominate the capacitance renormalization. Indeed, when $v$ is scaled with the amplitude $v_{0}$ (corresponding to the limit $N=\infty$ ), the leading exponential term reads as $v / \nu_{0} \sim \exp \left(N_{c} / N\right)$ with $N_{c}=\left(\pi^{2}+4\right) \sqrt{8 E_{J} / E_{C}}$. Here, $N_{c}$ is the typical length below which this finite-size correction becomes relevant. The behavior of the ratio $v / v_{0}$ is shown in Fig. 7.

The effective QPS amplitude $v$ decreases with increasing $N$. As a result, the parameter $q \sim N^{2} v(N)$ defined in Eq. (14) has a nonmonotonic behavior as a function of $N$. Accordingly, the maximum supercurrent obtained for Eq. (13) has also a nonmonotonic dependence on the total length $N$. In Fig. 8, we show the maximum supercurrent as a function of the ring size $N$ for different ratios of $E_{J} / E_{C}$. Decreasing $E_{J} / E_{C}$, the nonmonotonic behavior occurs at shorter lengths.

\section{B. Asymmetric effective potential $(q \gtrsim 1)$}

When the parameter $q$ is of order $\sim 1$, the maximum supercurrent for the model given by Eq. (13) shifts from the phase difference $\delta=\pi$ to the phase difference $\delta=\pi / 2$ (Fig. 5). The current-phase relation is strongly modified

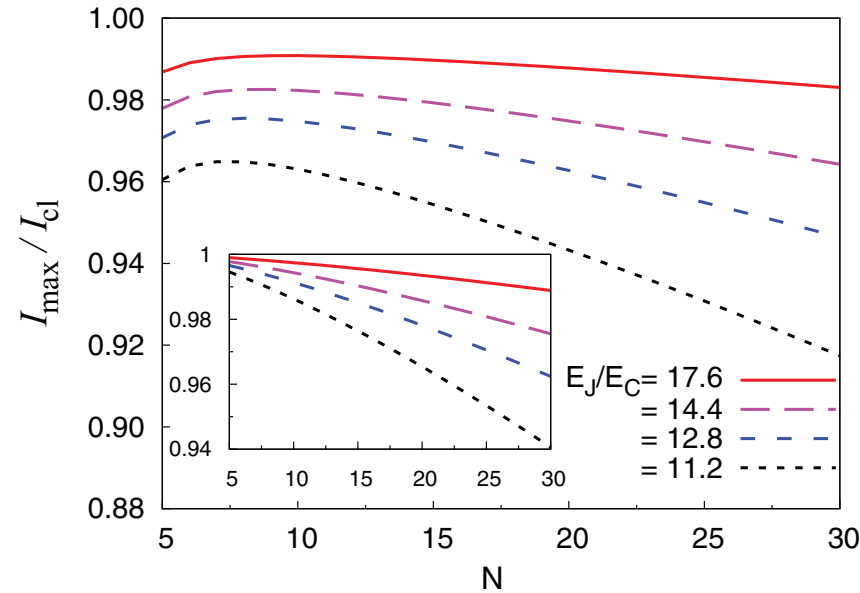

FIG. 8. (Color online) The maximum supercurrent $I_{\max }$ scaled with $I_{\mathrm{cl}}$ as a function of $N$ and at different values of the ratio $E_{J} / E_{C}$ by using the QPS amplitude $v(N)$ [Eq. (49)]. Inset: the maximum supercurrent by using the QPS amplitude $v_{0}$ [Eq. (1)].

passing from a sawtooth to a sinusoidal function. ${ }^{24}$ We should then solve Eq. (13) using the renormalized QPS amplitude $v_{\text {as }}$ associated with the full asymmetric effective potential $V_{\text {eff }}$ and which depends on $\delta$ (Fig. 4).

To reach the regime $q \gtrsim 1$, there are two possible ways. First, it can be reached by decreasing the ratio $E_{J} / E_{C}$ at given fixed length $N$, which is outside the range of validity of this work. Alternatively, for a given ratio $E_{J} / E_{C}$, we can increase the length $N$ of the system. The finite-size effects that we have discussed in the previous section for the phase difference $\delta=\pi$ vanish as $1 / N$. We now show that the finite-size corrections due to the asymmetry of the barrier vanish more rapidly, namely, as $1 / N^{2}$. Consequently, in the crossover range in which $N$ spans from $N_{\text {min }}=6$ to $N \gg 1$, we can neglect the difference between $\nu_{\text {as }}$ (for $\delta \neq \pi$ ) and $v$ (for $\delta=\pi$ ). It is then justified to use the renormalized amplitude $v$ for the symmetric potential (49) when calculating the maximum supercurrent for the model (13) in the full range of $q$.

Let us introduce $\kappa=1-\delta / \pi$ as the natural parameter to quantify the asymmetry. For an asymmetric potential, we can approximate the hopping energy between the two levels as the geometrical average obtained by considering the hopping for the left part of the potential and the right part of the potential with respect to the maximum ${ }^{40,41}$

$$
v \simeq \sqrt{v_{L} v_{R}}
$$

The left (right) amplitude $v_{L}\left(v_{R}\right)$ is the tunneling amplitude for the symmetric double potential (49), with the barrier height $2 E_{J}^{(+)}\left(2 E_{J}^{(-)}\right)$given by the difference between the maximum energy and the left (right) minimum. In a similar way, $\Delta \theta^{( \pm)} / 2 \pi$ is the distance between the maximum and the left (right) minimum point. Keeping the leading term of the expansion in $1 / N$, they read as $E_{J}^{( \pm)} / E_{J}=1-\left(\pi^{2} / 4 N\right) \pm$ $\left(\pi^{2} / 2 N\right) \kappa$ and $\Delta \theta^{( \pm)} / 2 \pi=1-1 / N \pm(\pi / N) \kappa$ where \pm is for $L$ and $R$. Inserting this expansion into Eq. (50), we finally conclude that finite-size corrections associated with the asymmetry parameter $\kappa$ cancel at order $1 / N$. When higher-order corrections are taken into account by using the full 
formula (50) for the QPS amplitude $v=v(\delta)$, the differences with the amplitude at $v=v(\pi)$ are practically unnoticeable.

Finally, our discussion is valid in the limit in which the asymmetry is sufficiently weak such that the two-level description remains valid. Close to the resonant condition, the excited and ground harmonic levels between two neighboring states in the effective potential (Fig. 4) are almost degenerate. This implies the inequality $E_{n}-E_{n+1}<\hbar \omega_{p}$ which translates into an interval for the magnetic flux $|\Delta \delta|<N \sqrt{2 E_{C} / E_{J}} / \pi$ around the degeneracy point at half a flux quantum. As long as the maximum supercurrent is well within this interval, our analysis based on the simple two-level description holds. Note that in the limit $N \rightarrow \infty$, the range $|\Delta \delta|$ is quite large as a consequence of the fact that $V_{\text {eff }}$ in Fig. 4 is practically indistinguishable from the periodic and multidegenerate cosine potential. On the other hand, for short rings, the maximum supercurrent occurs around $\delta \simeq \pi$ (Fig. 5), which is well inside the interval $|\Delta \delta|$ for a suitable choice of the parameters.

\section{EFFECTS OF THE CAPACITANCE $\boldsymbol{C}_{0}$}

We now consider the effect of the ground capacitance $C_{0}$ on the QPS amplitude. In view of the discussion of the previous section, we will focus on the analysis of the case $\delta=\pi$. Then, the effective potential appearing in the first line of Eq. (38) is replaced by a renormalized cosine potential

$$
\begin{aligned}
S_{\mathrm{eff}}= & \int_{0}^{\beta} d \tau\left[\frac{\hbar^{2}}{8 e^{2}}\left(C^{*}+\frac{C_{0}}{2}\right) \dot{\theta}^{2}(\tau)\right. \\
& \left.-E_{J}^{*} \cos \left(\frac{2 \pi}{\Delta \theta^{*}} \theta(\tau)\right)\right] \\
& +\frac{1}{2} \int_{0}^{\beta} d \tau \int_{0}^{\beta} d \tau^{\prime} G\left(\tau-\tau^{\prime}\right) \theta(\tau) \theta\left(\tau^{\prime}\right),
\end{aligned}
$$

where $C^{*}, E_{J}^{*}$ are defined in Eqs. (46) and (47) and $\Delta \theta^{*}$ in Eq. (48). Within the semiclassical instanton approach, ${ }^{42}$ the QPS amplitude reads as

$$
v=A \exp \left[-S_{\mathrm{eff}}^{(\mathrm{cl})} / \hbar\right]
$$

where $S_{\text {eff }}^{(\mathrm{cl})}$ is the effective action (51) evaluated at $\theta_{\mathrm{cl}}(\tau)$, the asymptotic path which minimizes the action and which connects the two relevant minima in the limit $\beta \rightarrow \infty$, i.e., the instanton solution. The prefactor $A$ is related to the quantum fluctuations around this minimum path. ${ }^{42}$ In contrast with the previous analysis for $C_{0}=0$, we now have to take into account the effects of the kernel $G(\tau)$ in the action (51).

\section{A. Parabolic approximation}

The first step is to find the classical path $\theta_{\mathrm{cl}}(\tau)$. For a cosine potential, this solution is known analytically only when the kernel is zero $G(\tau)=0\left(C_{0}=0\right)$. For the general case, to the best of our knowledge, the solution is unknown. We use the Villain approximation to solve the problem. ${ }^{43}$ We replace the periodic cosine potential by a parabolic potential

$$
\begin{aligned}
S_{p}= & \int_{-\frac{\beta}{2}}^{\frac{\beta}{2}} d \tau\left[\frac{\hbar^{2}}{8 e^{2}}\left(C^{*}+\frac{C_{0}}{2}\right) \dot{\theta}^{2}+\frac{V_{J}}{2} \min _{m}\left(\theta-m \Delta \theta^{*}\right)^{2}\right] \\
& +\frac{1}{2} \int_{-\frac{\beta}{2}}^{\frac{\beta}{2}} d \tau \int_{-\frac{\beta}{2}}^{\frac{\beta}{2}} d \tau^{\prime} G\left(\tau-\tau^{\prime}\right) \theta(\tau) \theta\left(\tau^{\prime}\right) .
\end{aligned}
$$

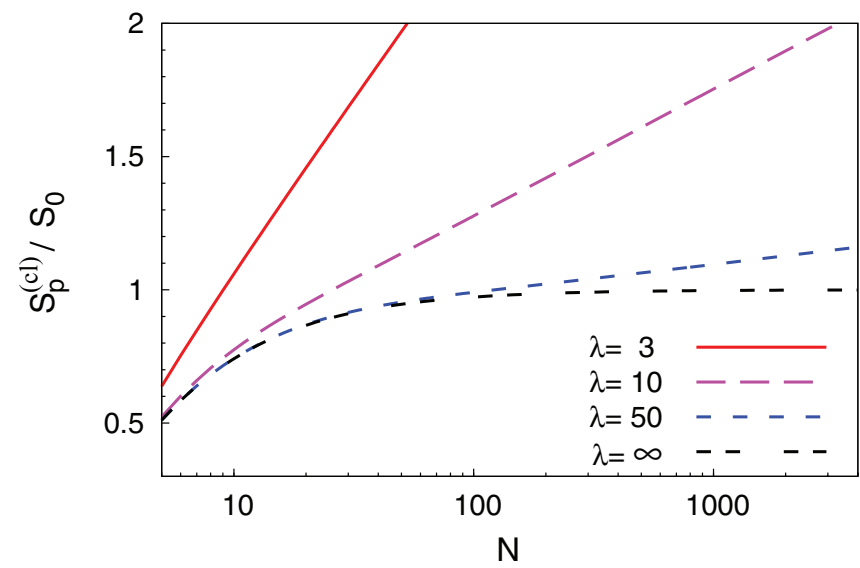

FIG. 9. (Color online) For $E_{J}=8 E_{C}$, the classical action $S_{p}^{(\mathrm{cl})}$ [Eq. (54)] scaled with $S_{0}=\left(8 E_{J} / E_{C}\right)^{1 / 2}$ as a function of $N$ for different screening lengths $\lambda=3,10,50, \infty$. Notice the crossover at $N \gtrsim \lambda$ to the logarithmic scaling $\sim \ln (N)$.

It is worth noting that, for $G=0\left(C_{0}=0\right)$ and $N \gg 1$, the instanton solution for this potential yields the action $S_{\mathrm{eff}}^{(\mathrm{cl})} / \hbar=$ $\pi^{2}\left(V_{J} / 8 E_{C}\right)^{1 / 2}$, i.e., the numerical coefficient is different from the one found for a cosine potential with the same amplitude $V_{J}$. Thus, in order to recover the previous results for $C_{0}=0$, it is convenient to set the height of the parabolic periodic potential to the value $V_{J}=\left(8 / \pi^{2}\right)^{2} E_{J}^{*}$ to take into account the difference between the profiles of the two potentials.

After the calculation (see Appendix B for details), the action (53) with the instanton path reads as ${ }^{44}$

$$
S_{p}^{(\mathrm{cl})}=2 \pi V_{J}\left(1-\frac{1}{N}\right)^{2} \int_{0}^{\infty} d \omega \frac{1}{\omega^{2}+\frac{4 e^{2} V_{J} / \hbar^{2}}{C^{*}+\frac{1}{2} C_{0}+\frac{4 e^{2}}{(\hbar \omega)^{2}} G(\omega)}},
$$

where $G(\omega)$ is the continuous limit for the Fourier transform $G\left(\omega_{\ell}\right)=G_{\ell}$ defined in Eq. (39). In Fig. 9, the behavior of $S_{p}^{(\mathrm{cl})}$ is shown as a function of the ring size $N$. For $C_{0}=0$ and $N \gg 1, S_{p}^{(\mathrm{cl})}$ saturates to $S_{0}=\left(8 E_{J} / E_{C}\right)^{1 / 2}$. When the ground capacitance is restored, we find a logarithmic scaling with $N$ for $N>\lambda$. Specifically, we find that $v \sim \exp [-\alpha \ln (N)]=$ $1 / N^{\alpha}$ with $\alpha=\pi \sqrt{E_{J} /\left(8 E_{0}\right)}$ (see Appendix C).

\section{B. Prefactor $\mathrm{A}$}

So far, our semiclassical approach was general. We will now restrict ourselves to the calculation of the finite-size corrections entering the renormalized amplitude $v$ with exponential accuracy; this corresponds to the leading dependence on $N$. Correspondingly, we will use an approximate expression for the prefactor $A$. This prefactor is associated with the quantum Gaussian fluctuations around the classical path. Specifically, we neglect the contribution of the low-energy paths having a mean kinetic energy lower than the height of the potential.

Proceeding as in Sec. IV D, to estimate the kinetic energy we determine the effective capacitance of the junction in the ring but now taking into account the finitesize effects. For $\omega_{\ell} \rightarrow \infty$, the kernel Eq. (39) reduces to 


$$
\begin{aligned}
& G_{\ell}=\Delta C\left(\hbar \omega_{\ell}\right)^{2} /\left(4 e^{2}\right) \text { where } \\
& \qquad \Delta C=\frac{C_{0}}{2(N-1)} \sum_{k=1}^{k_{\max }} \frac{1+\cos \left(\frac{2 \pi k}{N-1}\right)}{1-\cos \left(\frac{2 \pi k}{N-1}\right)+\frac{\pi^{2}}{2 \lambda^{2}}},
\end{aligned}
$$

so that the effective capacitance of the junction corresponds to

$$
C_{\text {eff }}=C^{*}+\frac{1}{2} C_{0}+\Delta C .
$$

Again, from Eq. (53), as $V_{J} \sim E_{J}$ we see that the threshold frequency separating the high- and low-energy regions is still $\omega_{\ell}=\omega_{\max }$ for $C>C_{0}$. From these observations, in order to take into account the contribution of the high-energy quantum fluctuations, we replace the effective capacitance in the prefactor $A$ of Eq. (49) (case $C_{0}=0$ ), with the effective capacitance Eq. (56). The result reads as

$$
A=\frac{4}{\sqrt{\pi}} \sqrt{\left(\frac{2 \pi}{\Delta \theta^{*}}\right)}\left(8 E_{J}^{* 3} \frac{e^{2}}{2 C_{\mathrm{eff}}}\right)^{\frac{1}{4}} .
$$

We now discuss the conditions for this approximation to be valid. For $\omega_{\ell}<\omega_{\max }$, the kernel $G$ in Eq. (53) couples the dynamics of the winding phase to the one of the modes. As we have seen at the end of Sec. IV D at $N=\infty$, for $C \gg C_{0}$ this interaction between the winding phase and the modes corresponds to a perturbation at low energies for the Gaussian harmonic fluctuations. Indeed, at $N=\infty$ we have weak damping for the quality factor $Q=2 \pi\left(C / C_{0}\right)^{1 / 2} \gg 1$. Although at finite $N$ the dynamics of the JJ ring does not correspond to a real resistance, the ratio $C_{0} / C$ still plays the role of a dimensionless coupling between the winding phase and the harmonic bath. The effect of this interaction on the low-energy Gaussian fluctuations can be neglected in the prefactor but not in the exponent where, as we have seen, they strongly affect the instanton classical path.

\section{Superconductor-insulator transition}

By inserting Eqs. (54) and (57) into (52), we obtain the QPS amplitude which we use to calculate the maximum supercurrent. Here, we discuss some numerical results valid in a general range of parameters, while in Appendix $C$ we discuss some analytic results valid for very long chains $(N \gg \lambda)$.

In Fig. 10, we plot the factor $q=N^{2} v(N) /\left(2 \pi^{2}\right)$ as a function of the ring size $N$ for the values $C=2 C_{0}$ and for different ratios of $E_{J} / E_{C}$. In the inset of Fig. 10, we also show the behavior of $v / E_{J}$. The first striking observation is that for large ratios of $E_{J} / E_{C}=2\left(E_{J} / E_{0}\right)$, the parameter $q$ does not increase as $N^{2}$ but, indeed, it vanishes upon increasing the length. This is due to the logarithmic dependence of the action $S_{\text {eff }}$ that we have obtained in Sec. VI A.

The presence of the logarithmic dependence is related to the lowest-energy modes (see Appendix C). Indeed, below the corresponding threshold $\omega_{\ell} \ll \omega_{\min }$, the winding junction feels the discreteness of the spectrum of the environment with which it can exchange energy. It is equivalent to say that there is no real dissipation at low frequency. An instanton solution conserving the initial energy still exists with a finite value of the corresponding action. For $N \gg \lambda$, we have $\omega_{\min } \sim$ $\left(8 E_{J} E_{0}\right)^{1 / 2} \pi / N$. Eventually, $\omega_{\min }$ vanishes as $N \rightarrow \infty$ and the action associated to the instanton diverges.

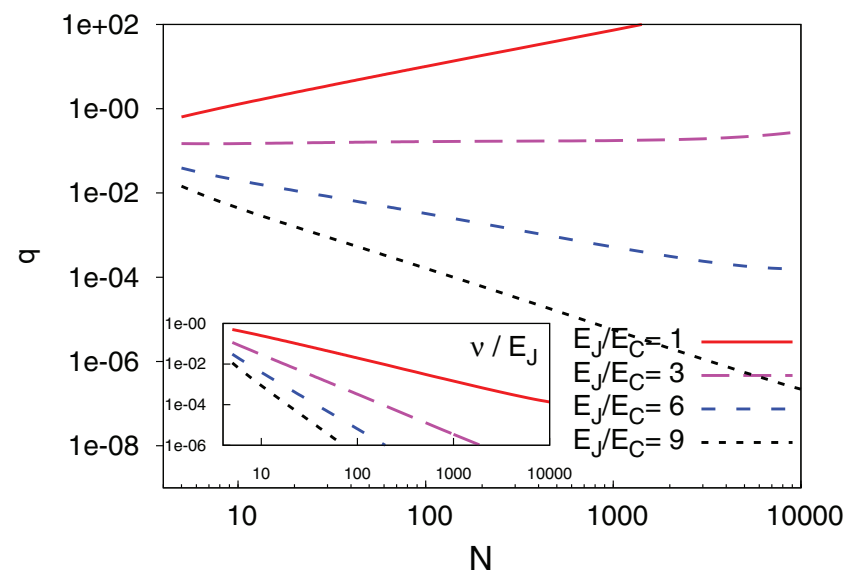

FIG. 10. (Color online) The parameter $q$ as a function of $N$ for $C=2 C_{0}$ and different ratios $E_{J} / E_{C}$. Inset: the behavior of the QPS amplitude $v$ scaled with $E_{J}$.

As a consequence, the parameter $q$ behaves as $q \sim N^{2} v \sim$ $N^{2-\alpha}$ and it scales either to zero or to infinity for $N=\infty$. A crossover is therefore expected at some critical ratio $E_{J} / E_{0}$ as $\alpha=\pi \sqrt{E_{J} /\left(8 E_{0}\right)}$ (see Appendix C).

The behavior of the maximum supercurrent $I_{\max }$ scaled with $I_{\mathrm{cl}}$ as a function of $N$ (Fig. 11) is related to the behavior of $q$. In the very long length limit, $I_{\max } / I_{\mathrm{cl}}$ increases and saturates to one for $E_{J} / E_{0}>3.24$, whereas for $E_{J} / E_{0}<3.24$ it vanishes. On the other hand, we remark that for finite systems, the current shows a nonmonotonic behavior: it increases with $N$ up to a maximum after which it decreases.

The interplay between finite-size effects, which scale as $v(N) \sim \exp \left(N_{c} / N\right)$, and the low-energy modes at finite $C_{0}$, which reduce the QPS amplitude as $v \sim 1 / N^{\alpha}$, cause that the total QPS amplitude of the ring $v_{\text {ring }}=N \nu(N)$ has a weak $N$ dependence so that the dependence of the maximum supercurrent on $N$ appears almost flat over a large range of $N$ when the critical ratio is approached (see Fig. 11).

Due to the nonmonotonicity of the current as a function of $N$, the critical point between the superconducting phase and

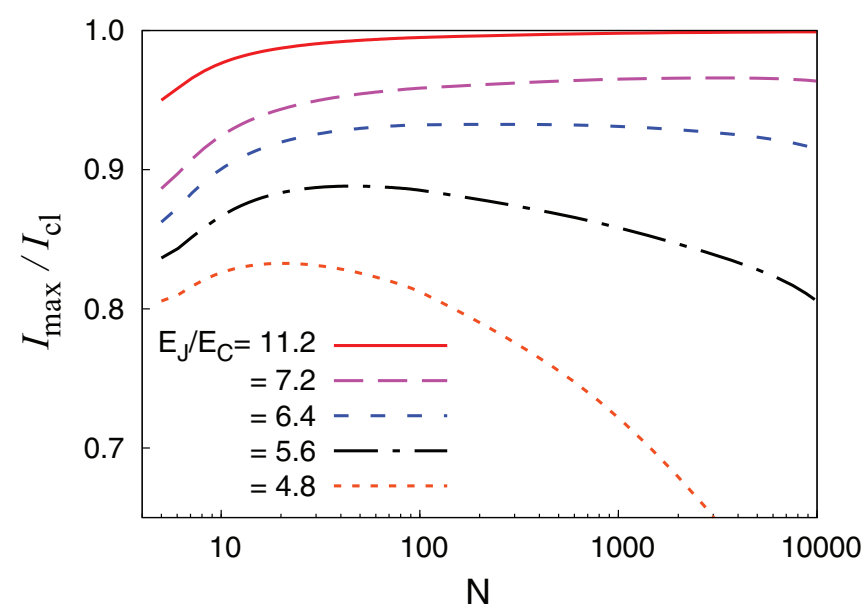

FIG. 11. (Color online) The maximum supercurrent $I_{\max }$ scaled with the classical value $I_{\mathrm{cl}}$ as a function of $N$ for $C=2 C_{0}$ and different ratios $E_{J} / E_{C}$. 


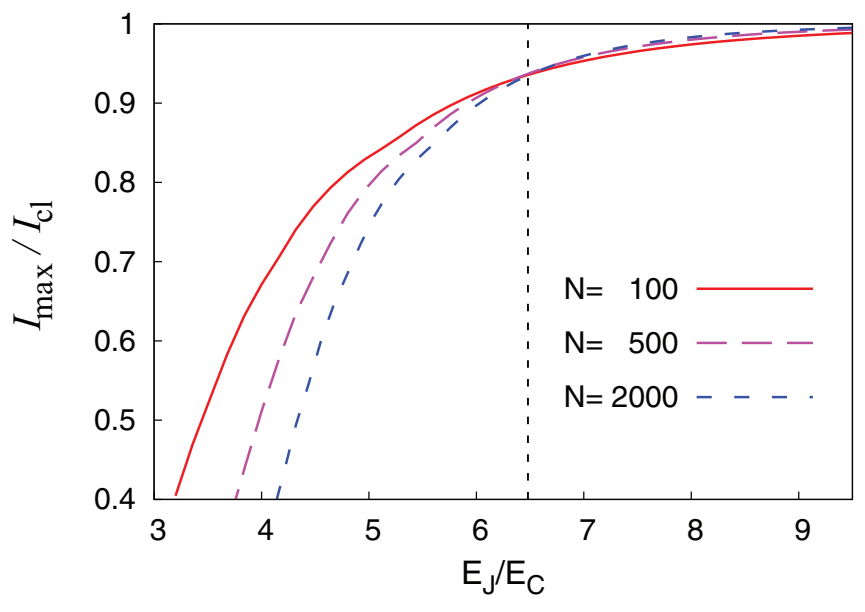

FIG. 12. (Color online) $I_{\max }$ scaled with $I_{\mathrm{cl}}$ as a function of $E_{J} / E_{C}=2 E_{J} / E_{0}$ for rings of different circumferences. The dashed vertical line corresponds to the critical value $E_{J} / E_{0}=32 / \pi^{2}$ of Ref. 15 for $C=2 C_{0}$.

the insulator phase can be better determined by plotting the maximal supercurrent as a function of the ratio $E_{J} / E_{C}$ for different circumferences. The result is shown in Fig. 12 for the case $C=2 C_{0}$.

We find that the critical value saturates to the value $E_{J} / E_{0} \simeq 3.24$, as long as $C \geqslant 2 C_{0}$. This result is in agreement with the result obtained by Korshunov ${ }^{15}$ who calculated $E_{J} / E_{0}=32 / \pi^{2}$ in the regime $C \gg C_{0}$. Reducing the ratio $C / C_{0}$, we find a small increase of the critical value $E_{J} / E_{0}$ which saturates to $\sim 4.16$ for vanishing mutual capacitance $C=0$. In this limit, we do not recover the critical ratio $E_{J} / E_{0}=128 / \pi^{2}$ obtained by Bradley and Doniach. ${ }^{13}$

\section{Discussion}

In this section, we discuss the validity of the single QPS approximation.

(a) The case $C_{0}=0$. In this case, it is most convenient to express the Lagrangian in terms of the phase differences $\left\{\theta_{n}\right\}$,

$$
\mathcal{L}=\sum_{n}\left[\frac{\hbar^{2} C}{8 e^{2}} \dot{\theta}_{n}^{2}-E_{J} \cos \left(\theta_{n}+\frac{\delta}{N}\right)\right],
$$

showing that there is no correlation in the spatial direction. Indeed, Eq. (58) with the constraint Eq. (3) describes $N-1$ independent variables. Under the condition $E_{J}>E_{C}$, one therefore can use the semiclassical instanton approach to describe the QPS in each individual junction. This is a wellcontrolled technique, ${ }^{42}$ based on the noninteracting (dilute) instanton approximation. For instance, in the limit $N \gg 1$, the problem reduces to the tunneling of a free particle in a double well. The resulting tunnel amplitude is $v_{0}$ [Eq. (1)], which is independent of the size of the system. The total amplitude $N v_{0}$ grows linearly with $N$ since instantons which can occur independently in any of the junctions, as previously analyzed in Ref. 24. The system becomes an insulator for $N \rightarrow \infty$; no phase transition occurs, in agreement with conclusions obtained in the thermodynamic limit. ${ }^{13-15}$

(b) The case $C_{0} \ll C$ and $N \lesssim \lambda$. When $C_{0}$ is restored, an interaction appears between the phase differences $\left\{\theta_{n}\right\} .{ }^{14}$
This interaction yields a possible coupling between QPSs occurring in different junctions $n \neq m$. This bare interaction is proportional to $C_{0} / C$. For finite systems and for $C_{0} \ll C$ we expect that, by continuity with the case $C_{0}=0$, this interaction can be neglected in first approximation. Indeed, the instantons are still rare events in the imaginary time for $E_{J} \gg E_{C}$ so that one can study a single instanton centered in one junction $\theta_{n_{0}}$. The other phase differences $n \neq N-1$ can be approximated by $N-2$ harmonic oscillators coupled to the winding phase $\theta_{n_{0}}$.

Another consequence of a finite $C_{0}$ is the fact that the modes have a frequency dispersion. This leads to an additional nonlocal term in the effective action of $\theta_{n_{0}}$ [Eq. (51)] beyond the finite-size corrections discussed for the case $C_{0}=0$. In particular, we considered the adiabatic regime in which all the modes have a frequency higher than the tunneling frequency of the phase $\theta_{n_{0}}$. This situation is very close to the case $C_{0}=0$ : the quantum tunneling of a fictitious particle in a double well can be still reduced to the tunneling between two levels but with an adiabatically renormalized amplitude. This is exactly the theoretical framework presented in Ref. 45, in which the authors developed the analysis for a generic two-level system. In particular, they considered the adiabatic regime expressed by their Eq. (2.9) which corresponds to our Eq. (52). We exploited this approach for a specific system, namely, the Josephson junction ring threaded by a magnetic flux. Moreover, as shown in Sec. VI and in Appendix C, the corrections to the instanton action due to the nonlocal term are small as long as $N \lesssim \lambda$. Therefore, by continuity to the previous case, we expect that our results are qualitatively and quantitatively correct in this regime.

(c) The case $C_{0} \ll C$ and $N \gg \lambda$. In the opposite limit $N \gg \lambda>1$ (see Appendix C), we find a logarithmic dependence on $N$ for the leading term in the instanton action $S_{p}^{(\mathrm{cl})} \sim \pi \sqrt{E_{J} /\left(8 E_{0}\right)} \ln (N / \lambda)$. Approaching the thermodynamic limit $(N \rightarrow \infty)$, even for $C_{0} \ll C$, the ground capacitance $C_{0}$ has a substantial effect. It leads to a renormalized QPS amplitude which strongly depends on the length of the JJ ring $v \sim v_{0} N^{-\sqrt{\pi^{2} E_{J} /\left(8 E_{0}\right)}}$. The validity of this result is addressed in the next section.

\section{E. Relation with the BKT transition}

Mathematically, the one-dimensional quantum model of the type in Eq. (2) can be mapped onto a two-dimensional classical model. Considering the axis $x=\tau$ (imaginary time) and the axis $y=n$ (position on the chain) for the local phases $\left\{\varphi_{n}(\tau)\right\}$, Bradley and Doniach showed that, for the case $C=0$, the 1D $\mathrm{JJ}$ chain is equivalent to anisotropic 2D classical spins with nearest-neighbor interactions along the perpendicular axis. ${ }^{13}$

Hence, according to this mapping, the superconductorinsulator phase transition occurring in the case $N=\infty$ corresponds to the order-disorder phase transition of an ensemble of ferromagnetically coupled planar classical spins. This is the celebrated Berezinskii-Kosterlitz-Thouless (BKT) transition, marked by a disruption of the ordered ferromagnetic phase due to the appearance of vorticity: topological defects for which the spin orientation changes by $2 \pi$ when following a closed path around them once. Reducing the ratio $E_{J} / E_{0}$, the BKT transition is driven by the dissociation of bound 
vortex-anti-vortex pairs formed in the ordered phase for the local phases $\left\{\varphi_{n}(\tau)\right\}$. $^{13}$ Thus, as interaction between vortices plays an essential role in this scenario, a natural question is the range of validity of our approach where the correlations between QPSs have been ignored.

Korshunov in Ref. 14 analyzed the same problem $(C=0)$ but using the representation in the space of the phase difference $\left\{\theta_{n}(\tau)\right\}$. In this space, the relevant (nonlinear) quantum fluctuations are the instantons, i.e., the QPSs. Notice that the vortices and the QPSs are not exactly the same object: the former are configurations defined in the space of the local phase $\left\{\varphi_{n}(\tau)\right\}$ of the BCS condensate in each superconducting island, ${ }^{13}$ whereas the latter are defined in the space of the phase differences $\left\{\theta_{n}(\tau)\right\}$ in each Josephson junction. ${ }^{14}$

The two instantons defined the $\theta$-space interact as ${ }^{14}$

$$
\mathcal{S}_{\text {int }}(\tau, \Delta N) \sim \pi \sqrt{E_{J} / E_{0}} \ln \left[\left(\omega_{0} \tau\right)^{2}+\Delta N^{2}\right],
$$

in which $\omega_{0} \sim\left(E_{J} E_{0}\right)^{1 / 2}$ and $(\tau, \Delta N)$ are the separation along the imaginary-time axis and in real space, respectively. Remarkably, two instantons interact again with a logarithmic potential in the two directions in a very similar way to the vortices defined the $\varphi$ space. This interaction leads to the formation of bound-pair states of QPSs which makes the superconducting phase stable, even for an infinite number of QPSs. This implies that, even if we focus on an individual junction $(\Delta N=0)$, interaction appears between different instantons along the imaginary time.

The crucial point is that this interaction is mediated by the propagating modes on the loop and is present only in the thermodynamic limit $N=\infty$, when the modes are sufficiently dense. ${ }^{45}$ In this limit, the local junction hosting a QPS is coupled to the rest of the infinite chain which acts as a dissipative environment. Indeed, the other Josephson junctions form a dense bath of harmonic oscillators with linear low-frequency dispersion. They mediate the interactions of two different instantons in imaginary time. This is the reason for the appearance of nonlocal correlations in time between different instantons. As discussed in Sec. IV D, the ratio $C_{0} / C$ plays the role of coupling between the winding phase and the harmonic bath of oscillators formed by the other $N-1$ junctions. Although the coupling constant is small $\left(C_{0} \ll C\right)$, the dynamics of the winding phase is now dissipative and the instantons in imaginary time are strongly coupled with a long-range logarithmic potential: the single QPS approximation breaks down.

As long as $N$ remains finite, no real dissipation appears and therefore no logarithmic interaction between instantons occurs in imaginary time. In particular, we studied the adiabatic regime in which the problem can be reduced to the renormalized quantum tunneling of a fictitious particle in a double-well potential ${ }^{45}$ (see the cases $b$ and $c$ in Sec. VID).

Between these two limits, we have a continuous crossover from the adiabatic regime to the full dissipative dynamics at $N=\infty$. Therefore, we expect that, at given ratio $C / C_{0}$ and $E_{J} / E_{C}$, there is a typical length $N^{*}$ which sets an upper bound for the range of validity of the single QPS approximation.

Indeed, increasing the length $N$, the lowest frequency $\omega_{\min }$ defined in Eq. (25) decreases as $\hbar \omega_{\min } \sim 4 \sqrt{2 E_{J} E_{C}}(\lambda / N)$. Then, for our approach to be self-consistent, we estimate $N^{*}$ as the point at which the adiabatic condition breaks down, namely, when the level splitting of the two-level system coincides with the lowest frequency of the modes: $2 v_{0} \sim \hbar \omega_{\min }$. It gives

$$
N^{*} / \lambda=\left(\sqrt{\pi} / 2^{3 / 4}\right) e^{\sqrt{8 \frac{E_{J}}{E_{C}}}} /\left(E_{J} / E_{C}\right)^{1 / 4} .
$$

As an example, at $E_{J} / E_{C}=6.5, \lambda=4.4\left(C=2 C_{0}\right)$ (the values close to the phase transition, see Fig. 12) we have the condition $N^{*} \sim 2800$.

For $N \gtrsim N^{*}$, only a finite number of discrete modes have frequencies lower than the tunneling frequency. ${ }^{46}$ Notice that this region beyond $N \gtrsim N^{*}$ is still far away from the full dissipative limit $N=\infty$, in which we expect a logarithmic interaction between instantons Eq. (59). Therefore, one has to consider Eq. (60) as a rough estimate. Remarkably, as shown in Fig. 12, in finite-size systems with $N \sim 10^{3}$ and well below the line $N=\infty$, the crossing point of the maximal current can be very close to the critical value of the phase transition although the current does not drop vertically to zero as expected in the real thermodynamic limit.

We conclude by recalling that the exact analysis of the interaction between two instantons in imaginary time beyond the limit $N \gg N^{*}$ in $\mathrm{JJ}$ chains is an interesting and open theoretical issue, beyond the aim of this work. Moreover, we remark that the Lagrangian of Eq. (16) can be exactly mapped on the classical (anisotropic) $X Y$ spin Hamiltonian, which represents the reference model for the BKT transition, only for $C=0$. For the general case of $\left(C, C_{0}\right)$, the twodimensional classical spin Hamiltonian associated with the Lagrangian of Eq. (16) has a four-body anisotropic-diagonal interaction between the spins $\varphi_{n}(\tau)$. Even at $N=\infty$, the superconductor/insulator transition in the extreme regime $C \gg C_{0}$ has not yet been analyzed in detail in the literature (with the exception of Ref. 15).

\section{SUMMARY AND CONCLUSIONS}

In this work, we studied the quantum QPS processes in 1D Josephson junction rings in the strong Josephson coupling limit $E_{J} \gg E_{C}, E_{0}$. In contrast with the previous work, ${ }^{24}$ we consider $\mathrm{JJ}$ rings of finite size in a wide range of lengths $(N \gtrsim 5)$ and with ground capacitance $C_{0}$. We calculated the renormalized QPS amplitude $v(N)$ and we discussed its consequence for the maximum supercurrent $I_{\max }$ flowing through a JJ ring threaded by a magnetic flux.

For the case $C_{0}=0$, we found an interplay between different finite-size effects which gives rise to a nonmonotonic behavior of the maximum supercurrent $I_{\mathrm{max}} / I_{\mathrm{cl}}$ as a function of the ring size. The critical length above which finite-size corrections are negligible is $N_{c} \sim\left(\pi^{2}+4\right) \sqrt{8 E_{J} / E_{C}}$.

When the ground capacitance is restored $C_{0}>0$, dispersive modes are possible on the ring which are directly coupled to the local winding phase difference. When $N \rightarrow \infty$, we found that the system converges either to a superconducting state with $I_{\max } / I_{\mathrm{cl}}=1$ or to an insulating phase $I_{\max } / I_{\mathrm{cl}}=0$, depending on the ratio $E_{J} / E_{0}$. For $C>C_{0}$, we found as critical ratio $\left(E_{J} / E_{0}\right)_{c}=3.24$, in agreement with the previous work of Ref. 15.

Although our analysis was mainly developed for a ring of identical junctions, it is also relevant for other systems, for 
instance, for a weak winding junction coupled to a chain of Josephson junctions in which the QPS is prevented, i.e., the so-called fluxonium. ${ }^{11}$ We have discussed the validity of the single QPS approximation. The regime $E_{C}>E_{J}$ associated with the regime of strong interaction between the QPSs is beyond the scope of this paper. A global phase diagram was reported some time ago in Refs. 14 and 15 in the thermodynamic limit, but it was based on a perturbative renormalization group analysis. A phase diagram obtained by nonperturbative approaches as well as the behavior of finite-size systems for arbitrary ranges of the parameters $E_{J}, E_{0}, E_{C}$, and $C / C_{0}$ constitutes a still open theoretical issue.

\section{ACKNOWLEDGMENTS}

We thank W. Guichard, L. Glazman, M. Vanević, L. Amico, and L. Ioffe for useful discussions. This work was supported by ANR through contracts DYCOSMA and QUANTJO. We acknowledge support from the European networks MIDAS, SOLID, and GEOMDISS and from Institut universitaire de France.

\section{APPENDIX A: PATH INTEGRAL}

In this Appendix, we summarize the main steps for the calculation of the path integral (36). First, we recall that any periodic function $\varphi_{n}$ on the lattice $n=0, \ldots, N-1$ can be decomposed as Eq. (18) where the set $\left\{\varphi_{k}\right\}$ are complex numbers, i.e., $\varphi_{k}=\varphi_{k}^{R}+i \varphi_{k}^{I}$. They are related by the condition $\varphi_{N-k}=\varphi_{k}^{*}$ as $\varphi_{n}$ is real. Using this property, we can write

$$
\varphi_{n}=\frac{1}{\sqrt{N}}[\varphi_{k=0}+\underbrace{(-1)^{n} \varphi_{k=\frac{N}{2}}}_{\text {N even }}+\sum_{k=1}^{k_{\max }}\left(\varphi_{k} e^{i \frac{2 \pi k}{N} n}+\text { c.c. }\right)] \text {. }
$$

For $N$ even we have $k_{\max }=(N / 2)-1$, whereas for $N$ odd we have $k_{\max }=(N-1) / 2$. We can express the Euclidean Lagrangians only in terms of the independent variables. Setting the action

$$
L_{0}\left(x_{k}\right)=\frac{1}{2} \mu_{k} \dot{x}_{k}^{2}+\frac{1}{2} \mu_{k} \omega_{k}^{2} x_{k}^{2},
$$

one can demonstrate that the harmonic Lagrangian (17) (omitting the constant proportional to $\delta_{m}$ ) in terms of the modes $k$ reads as

$$
L_{0}^{(N)}=\frac{\mu_{0}}{2} \dot{\varphi}_{k=0}^{2}+\underbrace{L_{0}\left(\varphi_{k=\frac{N}{2}}\right)}_{\text {N even }}+2 \sum_{k=1}^{k_{\max }}\left[L_{0}\left(\varphi_{k}^{R}\right)+L_{0}\left(\varphi_{k}^{I}\right)\right] .
$$

We now consider the actions (29), (31), and (32) where we have $N-1$ harmonic variables $\varphi_{n}$. Inserting Eq. (A1) with $N$ replaced by $N-1$ in Eq. (32), one can obtain that only the imaginary parts of the modes are linearly coupled to external forces $[\theta(\tau), \dot{\theta}(\tau)]$ through the position and through the velocity, i.e., Eq. (34). For the notation, we set

$$
q_{k}(\tau) \equiv \varphi_{k}^{I}(\tau)
$$

and we can write

$$
\mathcal{L}_{3}=\sum_{k=1}^{k_{\max }} \zeta_{k}\left(\frac{\hbar^{2} C}{8 e^{2}} \dot{q}_{k} \dot{\theta}+\frac{E_{J}}{2} q_{k} \theta\right) .
$$

From Eq. (A1) we observe that the phase $\varphi_{n=0}$ at $n=0$ depends only on the real part of the mode $\left\{\varphi_{k}^{R}\right\}$. Therefore, the first term of Eq. (31), $\varphi_{n=0}^{2}$, couples only the real parts of the different modes $k$. As real and imaginary parts are decoupled, the relevant term in Eq. (33) in which we are interested for the calculation of the effective action reduces to

$$
\mathcal{L}_{2} \sim \mathcal{E}_{0}+2 \sum_{k=1}^{k_{\max }}\left(\frac{1}{2} \mu_{k} \dot{q}_{k}^{2}+\frac{1}{2} \mu_{k} \omega_{k}^{2} q_{k}^{2}\right),
$$

with $\mathcal{E}_{0}=E_{J} \delta_{m}^{2}(N-1) /\left(2 N^{2}\right)$. We express the generic periodic path of the partition function as

$$
q_{k}(\tau)=q_{k, 0}+\sum_{\ell=1}^{+\infty}\left(q_{k, \ell} e^{i \omega_{\ell} \tau}+\text { c.c. }\right)
$$

in which the Fourier (Matsubara) component at $\omega_{\ell}=2 \pi \ell / \beta$ is given by $q_{k, \ell}=(1 / \beta) \int_{0}^{\beta} d \tau \exp \left(-i \omega_{\ell} \tau\right) q_{k}(\tau)$. Inserting the expression (A7) into the Lagrangian $\mathcal{L}_{2}+\mathcal{L}_{3}$ Eqs. (A5) and (A6) and integrating over the time, we obtain

$$
\int_{0}^{\beta} d \tau\left(\mathcal{L}_{2}+\mathcal{L}_{3}\right)=\mathcal{E}_{0}+2 \sum_{k=1}^{k_{\max }}\left(\mathcal{S}_{k, 0}+\sum_{\ell=1}^{+\infty} \mathcal{S}_{k, \ell}\right) .
$$

The first term $\mathcal{S}_{k, 0}$ contains only the component at zero frequency of $\theta(\tau)$ and $q_{k}(\tau)$ :

$$
\mathcal{S}_{k, 0}=\frac{\beta}{2} \mu_{k} \omega_{k}^{2} q_{k, 0}^{2}+\frac{\beta E_{J}}{2} q_{k, 0} \theta_{0} .
$$

The second terms $\mathcal{S}_{k, \ell}$ contain all the nonzero frequency components $(\ell>0)$

$$
\begin{aligned}
\mathcal{S}_{k, \ell}= & \beta \mu_{k}\left(\omega_{\ell}^{2}+\omega_{k}^{2}\right)\left|q_{k, l}\right|^{2} \\
& +\frac{\beta \zeta_{k} \hbar^{2}}{16 E_{C}}\left(\omega_{\ell}^{2}+\omega_{p}^{2}\right)\left(q_{k, \ell}^{*} \theta_{\ell}+\text { c.c. }\right) .
\end{aligned}
$$

Finally, the path integral is evaluated by integration over the Fourier components as

$$
\begin{aligned}
\Delta \mathcal{Z}= & \prod_{k=1}^{k_{\max }} \oint \mathcal{D}\left[q_{k}(\tau)\right] e^{-\frac{1}{\hbar} \int_{0}^{\beta} d \tau\left(\mathcal{L}_{2}+\mathcal{L}_{3}\right)}=e^{-\frac{\beta \mathcal{E}_{0}}{\hbar}} \\
& \times \prod_{k=1}^{k_{\max }} \int \frac{d q_{k, 0} e^{-\frac{2 \mathcal{S}_{k, 0}}{\hbar}}}{\sqrt{\frac{4 \pi \beta \hbar}{\mu_{k}}}} \prod_{\ell=1}^{+\infty} \iint \frac{d q_{k, \ell}^{R} d q_{k, \ell}^{I}}{\frac{\pi \hbar}{\beta \mu_{k} \omega_{\ell}^{2}}} e^{-\frac{2 \mathcal{S}_{k, \ell}}{\hbar}},
\end{aligned}
$$

where the preexponential factors are the Jacobians associated with the transformation of the path integral from the time space to the frequency space. ${ }^{42}\left(q_{k, \ell}^{R}, q_{k, \ell}^{I}\right)$ denote, respectively, the real and imaginary parts of $q_{k, \ell}$. The integral (A11) has the general Gaussian form and its evaluation is straightforward. 
After some algebra, the relevant exponential term is

$$
\Delta \mathcal{Z} \sim \exp \left\{\frac{\beta \hbar}{8 E_{C}} \sum_{\ell=0}^{\infty} Y\left(\omega_{\ell}\right) \frac{\left(\omega_{\ell}^{2}+\omega_{p}^{2}\right)}{1+\delta_{\ell, 0}}\left|\theta_{\ell}\right|^{2}\right\},
$$

with

$$
Y\left(\omega_{\ell}\right)=\frac{1}{N-1} \sum_{k=1}^{k_{\max }} \frac{\sin ^{2}\left(\frac{2 \pi k}{N-1}\right)}{1-\cos \left(\frac{2 \pi k}{N-1}\right)+\frac{\pi^{2}}{2 \lambda^{2}}\left(\frac{\omega_{\ell}^{2}}{\omega_{\ell}^{2}+\omega_{p}^{2}}\right)} .
$$

By adding the exponential term of Eqs. (A12) and (A13) to the Lagrangian $\mathcal{L}_{1}$ [Eq. (29)] and going back to the time representation, we obtain the result shown in Eqs. (37), (38), and (39).

\section{APPENDIX B: INSTANTON SOLUTION IN PERIODIC PARABOLIC POTENTIAL}

Referring to the action (53), we set $\hbar \omega_{J}=4 V_{J} e^{2} /$ $\left(C^{*}+C_{0} / 2\right)$ as a shorthand notation. The most general path can be always expressed as

$$
\theta(\tau)=\theta(-\beta / 2)+\int_{-\frac{\beta}{2}}^{\tau} d \tau^{\prime} \dot{\theta}\left(\tau^{\prime}\right)
$$

For the asymptotic instantonlike solution, we require the following boundary conditions at the end points $\theta_{i}=\theta(-\beta / 2)$ and $\theta_{f}=\theta(\beta / 2)$ :

$$
\lim _{\beta \rightarrow+\infty} \theta_{i}=0, \quad \lim _{\beta \rightarrow+\infty} \theta_{f}=\Delta \theta^{*} .
$$

It is useful to use the Fourier components of the velocity as free variables

$$
\dot{\theta}(\tau)=\sum_{\ell=-\infty}^{+\infty} \dot{\theta}_{\ell} e^{i \omega_{\ell} \tau}, \quad \dot{\theta}_{\ell}=\frac{1}{\beta} \int_{-\frac{\beta}{2}}^{\frac{\beta}{2}} d \tau \dot{\theta}(\tau) e^{-i \omega_{\ell} \tau}
$$

By assuming that the total energy is conserved, we impose that the initial velocity is equal to the final one: $\dot{\theta}(-\beta / 2)=\dot{\theta}(\beta / 2)$. More specifically, due to the symmetry of the potential, we can assume that the velocity is an even function of the time $\dot{\theta}(\tau)=\dot{\theta}_{0}+\sum_{\ell=1}^{+\infty} 2 \cos \left(\omega_{\ell} \tau\right) \dot{\theta}_{\ell}$, with $\dot{\theta}_{\ell}$ real numbers. By definition, the average velocity is $\dot{\theta}_{0}=\left(\theta_{f}-\theta_{i}\right) / \beta$. In terms of the variables $\left\{\dot{\theta}_{\ell}\right\}$, the path Eq. (B1) reads as

$$
\theta(\tau)=\theta_{i}+\dot{\theta}_{0}(\tau+\beta / 2)+\sum_{\ell \neq 0}\left(\frac{e^{i \omega_{\ell} \tau}-e^{-i \omega_{\ell} \frac{\beta}{2}}}{i \omega_{\ell}}\right) \dot{\theta}_{\ell} .
$$

We recall the definition of the kernel $G(\tau)$ in the action (53) in the Fourier space

$$
G(\tau)=\frac{1}{\beta} \sum_{\ell \neq 0} G_{\ell} e^{i \omega_{\ell} \tau}, \text { with } \quad G_{-\ell}=G_{\ell}^{*} .
$$

Using the expressions (B3), (B4), and (B5) in the action (53) and taking into account the symmetry respect to the time, the time integration is straightforward. The general action is thus expressed in terms of the variables $\left\{\dot{\theta}_{\ell}\right\}$ :

$$
\begin{aligned}
\frac{1}{\beta} S_{p}\left(\left\{\theta_{\ell}\right\}\right)= & \frac{V_{J}}{2 \omega_{J}^{2}} \dot{\theta}_{0}^{2}+\sum_{\ell=1}^{+\infty}\left(\frac{V_{J}}{\omega_{J}^{2}}+\frac{V_{J}}{\omega_{\ell}^{2}}+\frac{G_{\ell}}{\omega_{\ell}^{2}}\right) \dot{\theta}_{\ell}^{2} \\
& -\sum_{\ell=1}^{+\infty} \frac{4 V_{J}}{\beta \omega_{\ell}^{2}}\left[\theta_{i}\left(1-(-1)^{\ell}\right)+\frac{\beta \dot{\theta}_{0}}{2}\right] \dot{\theta}_{\ell} \\
& +\frac{V_{J}}{2}\left[\theta_{i}^{2}+\frac{\beta \dot{\theta}_{0}}{2} \theta_{i}+\frac{\left(\beta \dot{\theta}_{0}\right)^{2}}{12}\right] .
\end{aligned}
$$

Then, by the condition of minimization $\partial S_{p} / \partial \dot{\theta}_{\ell}=0$, we find the classical solution $\dot{\theta}_{\ell}^{(\mathrm{cl})}$ :

$$
\dot{\theta}_{\ell}^{(\mathrm{cl})}=\frac{2 \omega_{J}^{2} / \beta}{\omega_{\ell}^{2}+\omega_{J}^{2}+\frac{G_{\ell}}{V_{J}} \omega_{J}^{2}}\left[\left[1-(-1)^{\ell}\right] \theta_{i}+\frac{\beta \dot{\theta}_{0}}{2}\right] .
$$

We insert the solution (B7) into the action (B6) to obtain the value of the action at the minimum

$$
\frac{S_{p}^{(\mathrm{cl})}}{\hbar}=\frac{\beta V_{J} \dot{\theta}_{0}^{2}}{2 \hbar \omega_{J}^{2}}+\frac{4 V_{J}}{\hbar \beta} \sum_{\ell=1}^{+\infty} \frac{\left[\left[1-(-1)^{\ell}\right] \theta_{i}+\frac{\beta \dot{\theta}_{0}}{2}\right]^{2}}{\omega_{\ell}^{2}+\frac{\omega_{J}^{2}}{1+\omega_{J}^{2} G_{\ell} /\left(\omega_{\ell}^{2} V_{J}\right)}} .
$$

Finally, we take the limit $\beta \rightarrow \infty$. We use the boundary conditions (B2) and the fact that the average velocity $\dot{\theta}_{0}$ scales as $\left(\theta_{f}-\theta_{i}\right) / \beta \simeq \Delta \theta^{*} / \beta$. After that, the resulting series with respect to Matsubara frequencies $\ell=1, \ldots, \infty$ converges to an integral $\omega_{\ell}=\omega$ and the resulting action coincides with Eq. (54) of the main text by recalling $\hbar \omega_{J}=4 V_{J} e^{2} /$ $\left(C^{*}+C_{0} / 2\right)$ and Eq. (48) for $\Delta \theta^{*}$.

\section{APPENDIX C: LONG JJ RING WITH GROUND CAPACITANCE}

In this Appendix, we discuss some general features and some analytic limits of the effective action obtained within the parabolic approximation (54). To simplify the notation, we set $V_{J}=E_{J}$ as the results are qualitatively the same for two different coefficients. We focus on the long circumferences limit of the rings defined by the condition that we can neglect the corrections of order $(1 / N)$ in $E_{C}^{*}, E_{J}^{*}$, and $\Delta \theta^{*}$ as well as $N \gg \max (\lambda, 1)$. In this regime, we replace the sum with respect to the modes $k$ in $G\left(\omega_{\ell}\right) \equiv G(\omega)$ [Eq. (39)] with an integral and we obtain

$$
G(\omega)=\frac{(\hbar \omega)^{2}}{16 e^{2}} C_{0}\left(F(\omega) \sqrt{1+\frac{4 C}{C_{0}}}-1\right),
$$

where we set

$$
F(\omega)=\sqrt{1+\frac{\omega_{\max }^{2}}{\omega^{2}}}\left[1-\frac{2}{\pi} \arctan \left(\frac{\omega_{\min }}{\omega} \sqrt{1+\frac{\omega_{\max }^{2}}{\omega^{2}}}\right)\right],
$$

where $\omega_{\max }, \omega_{\min }$ are defined in Eqs. (22) and(25). Note that we can not neglect the $N$ dependence in the function $F(\omega)$ [Eq. (C2)], as it is strongly dependent on the minimum cutoff frequency $\omega_{\min }$. Using Eqs. (C1) and (C2), we can express the 
action on the classical instanton path Eq. (54) as

$$
\frac{S_{p}^{(\mathrm{cl})}}{\hbar}=2 \pi \frac{E_{J}}{\hbar} \int_{0}^{\infty} d \omega \frac{1}{\omega^{2}+\frac{\omega_{\max }^{2}}{1+2 F(\omega) /\left(1+4 C / C_{0}\right)^{1 / 2}}} .
$$

The two frequencies $\omega_{\max }, \omega_{\min }$ define three ranges for the integral on the frequency $\omega$. They are (i) $\omega_{\max } \ll \omega$, (ii) $\omega_{\min } \ll \omega \ll \omega_{\max }$, and (iii) $\omega \ll \omega_{\min }$. In these ranges, the function $F(\omega)$ can be approximated as (i) $F(\omega) \simeq 1$, (ii) $F(\omega) \simeq \omega_{\max } / \omega$, and (iii) $F(\omega) \simeq(N / \pi) /\left(1+4 C / C_{0}\right)^{1 / 2}$, to leading order in $1 / N$. Cutting the integral (C3) in three parts, we use the three approximated expressions for the function $F(\omega)$ to evaluate the integration

$$
\begin{aligned}
\int_{0}^{+\infty} d \omega \ldots= & \int_{\omega_{\max }}^{+\infty} d \omega \ldots+\int_{\omega_{\min }}^{\omega_{\max }} d \omega \ldots \\
& +\int_{0}^{\omega_{\min }} d \omega \ldots
\end{aligned}
$$

The first integral (i) in the high-frequency range as well as the third integral (iii) in the low-frequency range give a result independent of the ring's size $N$. On the other hand, for the second integral (ii) in the intermediate frequency range, we find the important result

$$
\begin{aligned}
\frac{S_{p}^{(\mathrm{cl})}}{\hbar} & \sim 2 \pi \frac{E_{J}}{\hbar} \int_{\omega_{\min }}^{\omega_{\max }} d \omega \frac{1}{\omega^{2}+\omega \omega_{\max }\left(1+4 C / C_{0}\right)^{1 / 2}} \\
& \sim \pi \sqrt{\frac{E_{J}}{8 E_{0}}} \ln \left(\frac{N}{\lambda}\right)+\ldots
\end{aligned}
$$

As we have reported numerically, we have a logarithmic dependence on $N$ of the classical action yielding to a power-law dependence of the QPS amplitude $v$. This leads to a superconductor/insulator phase transition when the limit $N=\infty$ is taken.

Finally, we observe that the function $F(\omega)$ [Eq. (C2)] saturates to a constant for $\omega \ll \omega_{\min }$ This low-frequency cutoff is important because it makes the total integral convergent. As we have explained in the text, below this threshold $\omega \ll \omega_{\min }$, the winding junction feels the discreteness of the spectrum of the environment with which it can exchange energy. That corresponds to say that there is no real dissipation at low frequency and an instanton solution conserving the initial energy still exists.
${ }^{1}$ L. B. Ioffe, M. V. Feigel'man, A. Ioselevich, D. Ivanov, M. Troyer, and G. Blatter, Nature (London) 415, 503 (2002).

${ }^{2}$ L. B. Ioffe and M. V. Feigel'man, Phys. Rev. B 66, 224503 (2002).

${ }^{3}$ B. Douçot and J. Vidal, Phys. Rev. Lett. 88, 227005 (2002).

${ }^{4}$ B. Douçot, M. V. Feigel'man, and L. B. Ioffe, Phys. Rev. Lett. 90, 107003 (2003).

${ }^{5}$ B. Douçot, M. V. Feigel'man, L. B. Ioffe, and A. S. Ioselevich, Phys. Rev. B 71, 024505 (2005).

${ }^{6}$ S. Gladchenko, D. Olaya, E. Dupont-Ferrier, B. Douçot, L. B. Ioffe, and M. E. Gershenson, Nat. Phys. 5, 48 (2009).

${ }^{7}$ M. A. Castellanos-Beltrana and K. W. Lehnert, Appl. Phys. Lett. 91, 083509 (2007).

${ }^{8}$ M. A. Castellanos-Beltrana, K. D. Irwin, G. C. Hilton, and L. R. Vale, Nat. Phys. 4, 928 (2008).

${ }^{9}$ W. Guichard and F. W. J. Hekking, Phys. Rev. B 81, 064508 (2010).

${ }^{10}$ J. Flowers, Science 306, 1324 (2004).

${ }^{11}$ V. E. Manucharyan, J. Koch, L. I. Glazman, and M. H. Devoret, Science 326, 113 (2009).

${ }^{12}$ C. Hutter, E. A. Tholén, K. Stannigel, J. Lidmar, and D. B. Haviland, Phys. Rev. B 83, 014511 (2011).

${ }^{13}$ R. M. Bradley and S. Doniach, Phys. Rev. B 30, 1138 (1984).

${ }^{14}$ S. E. Korshunov, Zh. Eksp. Teor. Fiz. 90, 2118 (1986) [Sov. Phys.JETP 63, 1242 (1986)].

${ }^{15}$ S. E. Korshunov, Zh. Eksp. Teor. Fiz. 95, 1058 (1989) [Sov. Phys.JETP 68, 609 (1989)].

${ }^{16}$ R. Fazio and H. van der Zant, Phys. Rep. 355, 235 (2001).

${ }^{17}$ S. Sarkar, Phys. Rev. B 75, 014528 (2007).

${ }^{18}$ S. Sarkar, Eur. Phys. J. B 67, 559 (2009).

${ }^{19}$ E. Chow, P. Delsing, and D. B. Haviland, Phys. Rev. Lett. 81, 204 (1998).

${ }^{20}$ D. B. Haviland, K. Andersson, and P. Agren, J. Low Temp. Phys. 118, 733 (2000).

${ }^{21}$ W. Kuo and C. D. Chen, Phys. Rev. Lett. 87, 186804 (2001).
${ }^{22}$ H. Miyazaki, Y. Takahide, A. Kanda, and Y. Ootuka, Phys. Rev. Lett. 89, 197001 (2002).

${ }^{23}$ Y. Takahide, H. Miyazaki, and Y. Ootuka, Phys. Rev. B 73, 224503 (2006).

${ }^{24}$ K. A. Matveev, A. I. Larkin, and L. I. Glazman, Phys. Rev. Lett. 89, 096802 (2002)

${ }^{25}$ I. M. Pop, I. Protopopov, F. Lecocq, Z. Peng, B. Pannetier, O. Buisson, and W. Guichard, Nat. Phys. 6, 589 (2010).

${ }^{26}$ T. P. Orlando, J. E. Mooij, L. Tian, C. H. van der Wal, L. S. Levitov, S. Lyod, and J. J. Mazo, Phys. Rev. B 60, 15398 (1999).

${ }^{27}$ K. K. Likharev and A. B. Zorin, J. Low Temp. Phys. 59, 347 (1985).

${ }^{28}$ G. Catelani, R. J. Schoelkopf, M. H. Devoret, and L. I. Glazman, Phys. Rev. B 84, 064517 (2011).

${ }^{29}$ Note that Eq. (1) corresponds to $\bar{\epsilon} / 2$ in Eq. (100) of Ref. 28.

${ }^{30}$ G.-L. Ingold, H. Grabert, and U. Eberhardt, Phys. Rev. B 50, 395 (1994).

${ }^{31}$ N. A. Masluk, I. M. Pop, A. Kamal, Z. K. Minev, and M. H. Devoret, Phys. Rev. Lett. 109, 137002 (2012).

${ }^{32}$ A. O. Caldeira and A. J. Leggett, Phys. Rev. Lett. 46, 211 (1981).

${ }^{33}$ A. Schmid, Phys. Rev. Lett. 51, 1506 (1983).

${ }^{34}$ Note that the model discussed here is different from the one introduced by Z. Hermon, E. Ben-Jacob, and G. Schön, Phys. Rev. B 54, 1234 (1996), in which the kinetic inductance of the superconducting grains was assumed to be much larger than the Josephson inductance. Here, we consider the experimentally more relevant opposite regime where the Josephson inductance dominates (see also Ref. 35).

${ }^{35}$ F. W. J. Hekking and L. I. Glazman, Phys. Rev. B 55, 6551 (1997).

${ }^{36} \mathrm{M}$. Tinkham, Introduction to Superconductivity, 2nd ed. (McGrawHill, Singapore, 1996).

${ }^{37}$ I. M. Pop, B. Douçot, L. Ioffe, I. Protopopov, F. Lecocq, I. Matei, O. Buisson, and W. Guichard, Phys. Rev. B 85, 094503 (2012). 
${ }^{38}$ The detailed comparison of our result with Eq. (8) of Ref. 15 requires the substitution $E_{J} \rightarrow \hbar V$ and $\hbar^{2} C /\left(4 e^{2}\right)^{\sim} \rightarrow M, \hbar^{2} C_{0} /$ $\left(4 e^{2}\right) \rightarrow m$.

${ }^{39}$ G. Schön and A. D. Zaikin, Phys. Rep. 198, 237 (1990).

${ }^{40}$ G. Rastelli, Phys. Rev. A 86, 012106 (2012).

${ }^{41}$ P. R. Johnson, W. T. Parsons, F. W. Strauch, J. R. Anderson, A. J. Dragt, C. J. Lobb, and F. C. Wellstood, Phys. Rev. Lett. 94, 187004 (2005); J. M. Schmidt, A. N. Cleland, and J. Clarke, Phys. Rev. B 43, 229 (1991).

${ }^{42}$ H. Kleinert, Path Integral in Quantum Mechanics, Statistics and Polymer Physics, 2nd ed. (World Scientific, Singapore 1995).

${ }^{43}$ J. Villain, J. Phys. (Paris) 36, 581 (1975).
${ }^{44}$ Note that for $G(\omega)=0\left(C_{0}=0\right)$, the integral converges to the factor $\pi / 2$ and we obtain $S_{\mathrm{par}} / \hbar=8\left[\Delta \theta^{*} /(2 \pi)\right]^{2} \sqrt{E_{J}^{*} / E_{C}^{*}}$. Actually, this result is slightly different from the one obtained with the cosine potential Eq. (49). In the latter case, the classical action depends linearly on $\Delta \theta^{*} /(2 \pi)=1-1 / N$. At finite length, finite-size corrections in $1 / N$ carry a different numerical prefactor.

${ }^{45}$ A. J. Leggett, S. Chakravarty, A. T. Dorsey, M. P. A. Fisher, A. Garg, and W. Zwerger, Rev. Mod. Phys. 59, 1 (1987).

${ }^{46}$ Notice that, when we consider the regimes $C_{0}>0$ and $N \gg \lambda$, the condition of validity $N<N^{*}$ also implies an upper bound to the parameter $q$ defined in Eq. (14). 